TOPICAL REVIEW

\title{
The role of phonons for exciton and biexciton generation in an optically driven quantum dot
}

\author{
D E Reiter ${ }^{1}$, T Kuhn ${ }^{1}, \mathrm{M} \mathrm{Glässl}^{2}, \mathrm{~V}$ M Axt ${ }^{2}$ \\ ${ }^{1}$ Institut für Festkörpertheorie, Universität Münster, Wilhelm-Klemm-Straße \\ 10, 48149 Münster, Germany \\ 2 Theoretische Physik III, Universität Bayreuth, 95440 Bayreuth, Germany
}

\begin{abstract}
For many applications of semiconductor quantum dots in quantum technology a well controlled state preparation of the quantum dot states is mandatory. Since quantum dots are embedded in the semiconductor matrix, the interaction with phonons plays often a major role in the preparation process. In this review, we discuss the influence of phonons on three basically different optical excitation schemes which can be used for the preparation of exciton, biexciton, and superposition states: a resonant excitation leading to Rabi rotations in the excitonic system, an excitation with chirped pulses exploiting the effect of adiabatic rapid passage, and an off-resonant excitation giving rise to a phononassisted state preparation. We give an overview over experimental and theoretical results showing the role of the phonons and compare the performance of the schemes for state preparation.

PACS numbers: 78.67.Hc, 63.20.kd, 78.47.-p, 42.50.Hz
\end{abstract}

\section{Introduction}

Quantum dots (QDs) are semiconductor nanostructures that combine aspects typical for atomic systems, in particular a discrete energy spectrum, with the technologically well developed and well controllable semiconductor material system. This compatibility with existing technologies makes QDs attractive candidates for a wide range of applications ranging from optoelectronic devices like QD lasers [1, 2, 3, 4, where the target is an improved performance compared to other laser structures, up to new applications in the fields of quantum cryptography or quantum information processing, where the presence of discrete energy levels is mandatory. Examples of such quantum applications are single-photon sources [5, 6, 7, 8, 9, 10, sources of entangled photon pairs [11, 12, 13, 14, 15, 16, 17, 18, and qubit devices or quantum gates [19, 20, 21, 22, 23, 24]. The functionality of all these latter applications relies on the preparation of a well-defined quantum state. The generation of single photons or entangled photon pairs is based on the radiative recombination starting from the exciton or the biexciton state. Therefore, for a highly efficient photon generation a high-fidelity preparation of the QD in the exciton or biexciton state is required. For the realization of a qubit or a quantum gate in addition arbitrary superposition states have to be prepared with high fidelity.

In an ideal optically driven two- or few-level system a preparation of arbitrary quantum states provides no conceptual difficulty and can be achieved, e.g., by using various coherent control techniques [25. A QD, however, due to its embedding in 
a semiconductor matrix and the resulting non-negligible coupling to the phonons of the crystal lattice, is not an ideal few-level system. Phonons lead to a dephasing of coherences and to relaxation processes between different electronic states. This may prohibit some of the preparation schemes or at least strongly limit the range of excitation parameters where a high-fidelity state preparation is possible. Therefore, a detailed knowledge of the role of phonons in the various excitation schemes is necessary to select the best scheme and to optimize its performance.

In this review we will give an overview of the role of phonons in different state preparation schemes using optical excitation of a single QD. In particular, we will discuss the phonon influence on coherent excitation schemes based on resonant excitation or excitation by chirped laser pulses, where phonons typically give rise to unwanted dephasing or relaxation effects and therefore introduce limitations to the otherwise ideal schemes. In contrast to these coherent schemes, phonons may also be actively used in state preparation by employing an excitation with detuned pulses giving rise to a phonon-assisted exciton or biexciton generation.

The review is organized as follows. In Sec. 2 the model for the description of the electronic structure of the QD as well as its coupling to light and phonons is introduced. The relevant physics and the different theoretical approaches which have been used to model the dynamics of the optically driven QD are briefly explained. In Sec. 3 the three basically different state preparation schemes are described. Experimental and theoretical results that have been obtained with these schemes are discussed in particular in view of the role of the phonons for the state preparation. In Sec. प we compare the three schemes and discuss their respective advantages, drawbacks, and limitations. The review then finishes with some concluding remarks.

\section{Theoretical background}

\subsection{Quantum dot model}

In QDs the electronic motion is confined in all three directions on a nanometer scale 1. 26, 27. Therefore, the corresponding electronic spectra possess a discrete part similar to what is found for atoms. The target states of the preparation schemes to be discussed in this review are typically well separated in energy from all other electronic states, which allows for a selective excitation of these states. This condition is best fulfilled for strongly confined dots. For the theory, this implies that one can concentrate on a rather limited electronic subspace spanned by states $|\nu\rangle$ with discrete energies $\hbar \omega_{\nu}$ such that the electronic Hamiltonian reads:

$$
H_{\mathrm{dot}}=\sum_{\nu} \hbar \omega_{\nu}|\nu\rangle\langle\nu| .
$$

Most often discussed is the case, where the valence band states are formed from heavyhole states with angular momentum projections $J_{z}= \pm 3 / 2$ while the conduction band states have $J_{z}= \pm 1 / 2$. For strongly confined dots, one can concentrate on the lowest lying electron and hole states. In this review we will restrict ourselves to the case of charge-neutral QDs, which implies that one has to consider a basis of four electronic states, namely the ground state $|G\rangle$ (i.e., the state without electron-hole pairs), two bright single exciton states $\left|X_{ \pm}\right\rangle$corresponding to single electron-hole pairs with total angular momenta \pm 1 and the biexciton state $|B\rangle$, where two electron-hole pairs are confined in the dot. Two further exciton states with angular momenta \pm 2 can be formed from the lowest single particle states, which are usually referred to as dark 
excitons as they cannot be excited directly by the laser field. A relaxation into these dark states requires spin flip processes that take place on a time scale of typically longer than a nanosecond [28, 29, 30] or relaxation from energetically higher excited states 31. Therefore, they are not relevant for state preparation schemes occurring on a time scale of at most a few tens of picoseconds and will not be considered in this review.

In most real QDs the two bright exciton states exhibit an additional coupling due to the long-range exchange interaction:

$$
H_{\text {exchange }}=V_{\text {ex }}\left(\left|X_{+}\right\rangle\left\langle X_{-}|+| X_{-}\right\rangle\left\langle X_{+}\right|\right) \text {. }
$$

A finite $V_{\text {ex }}$ entails a splitting of the exciton energies by $2\left|V_{\text {ex }}\right|$ and the exciton eigenstates are then given by the linearly polarized single exciton states:

$$
\left|X_{x}\right\rangle=\frac{1}{\sqrt{2}}\left(\left|X_{+}\right\rangle+\left|X_{-}\right\rangle\right), \quad\left|X_{y}\right\rangle=\frac{i}{\sqrt{2}}\left(\left|X_{+}\right\rangle-\left|X_{-}\right\rangle\right) .
$$

The size of the exchange splitting strongly depends on the dot geometry. Typical values in InGaAs QDs range from almost zero to a few $100 \mu \mathrm{eV}$ [32, 33. For schemes that are targeted at the preparation of the biexciton state, in order to subsequently initiate a decay cascade that creates entangled photon pairs [12, 15, 17] it is a necessary precondition to ideally have a vanishing exchange splitting [15, 16, 34, 35]. Otherwise, a kind of which-path information would be introduced in the decay that prevents a high degree of entanglement [14, 36]. Apart from selecting QDs that happen to exhibit almost zero splitting [16], also specially designed growth [37] or annealing [38] techniques have been developed for that purpose. Alternatively, one can use dots with high symmetry such as, e.g., self-organized In(Ga)As/GaAs QDs grown on (111) substrate which should ideally have no exchange splitting [39, or apply strategies to actively suppress the splitting, such as, e.g., the ac-Stark tuning [40] or application of strain or external electric and/or magnetic fields [41, 42, 43, 44, 45, 46. Furthermore, all preparation schemes aim at being as fast as possible without degrading other properties such as the robustness or the spectral selectivity of the scheme. With the schemes discussed in this review the preparation is typically completed after about $4-20$ ps. Together with the fact that preparation schemes are preferably executed with dots having a rather small exchange splitting, it is justified to ignore the impact of the exchange interaction on the time scale required for the preparation. Therefore, in the following we shall identify the relevant dot eigenstates with the states: $|G\rangle$, $\left|X_{ \pm}\right\rangle$and $|B\rangle$, i.e., we have two energetically degenerate single exciton states with energy $\hbar \omega_{X}$. When the energy of the ground state is set to zero, the energy of the biexciton state $\hbar \omega_{B}$ is given by $\hbar \omega_{B}=2 \hbar \omega_{X}-\Delta_{B}$, where $\Delta_{B}$ denotes the biexciton binding energy.

The external driving of the system by a coherent laser field is commonly modeled by the dipole coupling to a classical light field in the rotating wave approximation corresponding to the Hamiltonian:

$$
H_{\mathrm{dot}-\mathrm{laser}}=-\sum_{\nu \nu^{\prime}} \hbar M_{\nu \nu^{\prime}}|\nu\rangle\left\langle\nu^{\prime}\right|,
$$

where $M_{\nu \nu^{\prime}}$ is the matrix of dipole interactions. Accounting for the usual dipole selection rules between the dot basis states, the dipole interaction matrix $M$ reads:

$$
M=\frac{1}{2}\left(\begin{array}{cccc}
0 & \Omega_{\sigma^{+}}^{*}(t) & \Omega_{\sigma^{-}}^{*}(t) & 0 \\
\Omega_{\sigma^{+}}(t) & 0 & 0 & \Omega_{\sigma^{-}}^{*}(t) \\
\Omega_{\sigma^{-}}(t) & 0 & 0 & \Omega_{\sigma^{+}}^{*}(t) \\
0 & \Omega_{\sigma^{-}}(t) & \Omega_{\sigma^{+}}(t) & 0
\end{array}\right),
$$




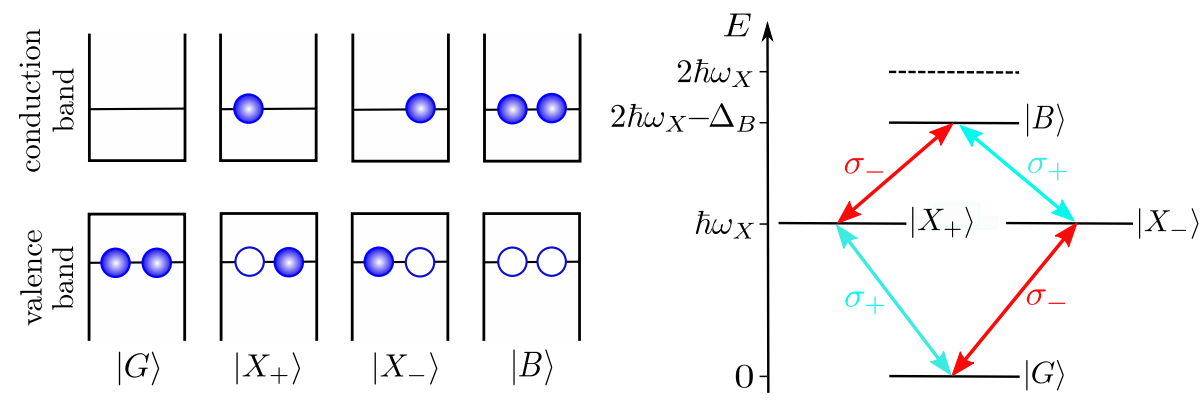

Figure 1. Electronic levels and excitation-level diagram for an exciton-biexciton QD system in the case of a vanishing exchange interaction. $|G\rangle$ denotes the ground state without electron-hole pairs, $\left|X_{ \pm}\right\rangle$are two single exciton states of different angular momentum, which can be excited separately by $\sigma^{ \pm}$circularly polarized light, and $|B\rangle$ is the biexciton state. In the left figure, filled circles represent electrons, whereas open circles represent holes. In the right figure, $\hbar \omega_{X}$ denotes the ground state to exciton transition energy and $\Delta_{B}$ is the biexciton binding energy.

where $\Omega_{\sigma^{ \pm}}(t) \equiv f_{\sigma^{ \pm}}(t) e^{-i \omega_{L} t}$ with $f_{\sigma^{ \pm}}(t)=2 M_{0} E_{\sigma^{ \pm}}(t) / \hbar$. Here, $E_{\sigma^{ \pm}}(t)$ is the circularly $\sigma^{ \pm}$polarized component of the laser field which drives the dipole transitions between the ground state $|G\rangle$ and the single exciton states $\left|X_{ \pm}\right\rangle$as well as from the single exciton states $\left|X_{\mp}\right\rangle$ to the biexciton state $|B\rangle, M_{0}$ denotes the corresponding dipole matrix element, $\omega_{L}$ is the central frequency of the laser, and $f_{\sigma^{ \pm}}(t)$ is the driving amplitude of the corresponding transition, which is defined in such a way that for a resonant $\mathrm{cw}$ excitation its modulus corresponds to the Rabi frequency. This coupling scheme is illustrated in Fig. 1]

In many studies, this model is further reduced to an electronic two-level system (TLS) by considering an excitation by light with a single circular polarization. For this choice of excitation conditions, two of the four electronic states introduced above are decoupled from the dynamics and the electronic system is reduced to the TLS formed by the ground state $|G\rangle$ and the single exciton state that we shall denote by $|X\rangle$ driven by a light field with the amplitude $f$.

For laser pulse envelopes that are varying sufficiently slowly in time, the dressed state basis for the electronic state space is a natural choice, which also helps to highlight the physics of some of the preparation schemes discussed in Sec. 3 These dressed states are defined as the instantaneous eigenstates of the light-matter Hamiltonian $H_{\text {dot }}+H_{\text {dot-laser }}$ in the frame rotating with the laser frequency. The simplest case is constituted by the TLS, where the dressed states $\left|D_{ \pm}\right\rangle$are given by:

$$
\left|D_{ \pm}\right\rangle=N_{ \pm}\left[f^{*}|G\rangle+\left(\Delta \mp \sqrt{\Delta^{2}+|f|^{2}}\right)|X\rangle\right],
$$

where $\Delta$ is the detuning between the laser frequency and the transition frequency of the dot, and $N_{ \pm}$are normalization factors. The energies of the dressed states are

$$
\hbar \Omega_{ \pm}=\frac{\hbar}{2}\left(-\Delta \pm \sqrt{\Delta^{2}+|f|^{2}}\right) .
$$

As can be seen from these formulas, the dressed states depend on the detuning $\Delta$ and the strength $f$ of the optical driving. For a four-level exciton-biexciton system, simple formulas for the dressed states arise in the special case of a vanishing biexciton binding energy $\Delta_{B}$ and resonant driving [47. For finite $\Delta_{B}$ and/or off-resonant driving 
analytical expressions can still be derived, but they are quite involved and not very instructive.

Due to their embedding in a solid-state environment, QDs cannot be treated as isolated systems. Here, most important is the coupling to phonons. For strongly confined dots, on short time scales, the coupling of excitons to acoustic phonons via the pure dephasing mechanism [48, 49, 50, 51] has been identified as typically being the dominant source of decoherence [50, 52, 53, 54. The corresponding Hamiltonian reads:

$$
H_{\text {dot-phonon }}=\sum_{\mathbf{q}} \hbar \omega_{\mathbf{q}} b_{\mathbf{q}}^{\dagger} b_{\mathbf{q}}+\sum_{\mathbf{q}, \nu} \hbar\left(g_{\mathbf{q}}^{\nu} b_{\mathbf{q}}+g_{\mathbf{q}}^{\nu *} b_{\mathbf{q}}^{\dagger}\right)|\nu\rangle\langle\nu| .
$$

The operator $b_{\mathbf{q}}^{\dagger}\left(b_{\mathbf{q}}\right)$ creates (annihilates) an acoustic phonon in a mode labeled by $\mathbf{q}$, and $\hbar \omega_{\mathbf{q}}$ is the corresponding phonon energy. In this review we will mainly concentrate on strongly confined self-assembled InGaAs or GaAs QDs, where the lattice properties in- and outside the dot are similar. Therefore, the phonon modes can be approximated by bulk modes, where the label $\mathbf{q}$ denotes the phonon wave vector. Furthermore, for strongly confined dots, the excitonic and biexcitonic wave functions approximately factorize into products of single particle electron and hole wave functions, which implies that the phonon coupling constants are of the form: $g_{\mathbf{q}}^{\nu}=n_{\nu} g_{\mathbf{q}}$, where $n_{\nu}$ represents the number of excitons present in the state $|\nu\rangle$ and $g_{\mathbf{q}}$ is the exciton-phonon coupling [51. The explicit form of $g_{\mathbf{q}}$ depends on the specific coupling mechanism. For typical InGaAs or GaAs dots, as discussed mostly in this review, the deformation potential coupling to longitudinal acoustic (LA) phonons is by far the strongest, while for strongly polar materials like GaN or QDs with spatially strongly separated electron and hole wave functions, the piezoelectric coupling to both longitudinal and transverse phonons may become dominant [51, 55, 56].

The carriers in the QD also interact with optical phonons, e.g., via the Fröhlich interaction [57, 58, 59, 60, which under certain conditions can also contribute to the generation of biexcitons 61. In contrast to acoustic phonons, optical phonons, in particular those which are efficiently coupled to the electrons and holes in a QD, can typically be taken to be dispersionless. Because of their essentially discrete spectrum, the interaction with optical phonons does not give rise to dephasing, instead it leads to an oscillatory contribution to the dynamics of the polarization visible as discrete sideband in the optical spectrum 51, 62. However, the interaction strength of the energetically well separated optical phonons compared to acoustic phonons is typically rather small and except for very short pulse excitations they are usually out of the spectral range of excitation. Therefore, we will focus on the acoustic phonons in the remainder of this review. Explicit expressions for all commonly used carrier-phonon coupling schemes for QDs can be found in Ref. [51].

Most important in the context of state preparation is the influence of the phonon degrees of freedom on the electronic dynamics. The latter is determined by the phonon spectral density $J(\omega)$, which is defined by:

$$
J(\omega)=\sum_{\mathbf{q}}\left|g_{\mathbf{q}}\right|^{2} \delta\left(\omega-\omega_{\mathbf{q}}\right) .
$$

For QDs with a harmonic confinement potential in which the carriers are coupled to LA phonons via the deformation potential the phonon spectral density is well approximated by [63, 64]:

$$
J(\omega)=A \omega^{3} \exp \left(-\omega^{2} / \omega_{c}^{2}\right)
$$




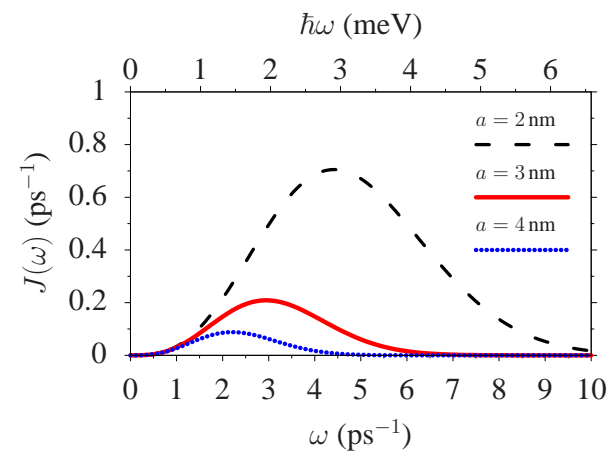

Figure 2. Phonon spectral density for different localization lengths of the QD wave functions.

with a strength $A$ and a cut-off frequency $\omega_{c}$ determined by the size of the QD. For a spherical QD with equal confinement lengths $a$ for electrons and holes this formula is exact and the parameters are given by

$$
A=\frac{\left|D_{e}-D_{h}\right|^{2}}{4 \pi^{2} \varrho \hbar c_{s}^{5}} \quad \text { and } \quad \omega_{c}=\sqrt{2} \frac{c_{s}}{a},
$$

where $D_{e / h}$ are the deformation potentials of electrons and holes, $\varrho$ is the crystal density, and $c_{s}$ is the longitudinal sound velocity. Figure 2 shows $J(\omega)$ for three different dot sizes and GaAs parameters. It is seen that $J(\omega)$ is maximal at a finite phonon frequency, which is larger for smaller dots and typically of the order of a few $\mathrm{meV}$. For small frequencies the spectral density scales according to $J(\omega) \sim \omega^{3}$. Such a power-law behavior with an exponent $n>1$ is referred to as superohmic coupling 65.

The smooth phonon spectral density of Fig. 2 is typical for the interaction of excitons or biexcitons in a single QD with bulk acoustic phonon modes, where the size of the QD is the only characteristic length scale. Additional structures in the spectral density appear as soon as other characteristic length scales are present in the system. Phonon couplings for various types of QD structures with more than one length scale have been discussed in the literature, such as QDs with different confinement lengths of electrons and holes [66] or with different aspect ratios [67, strong variations of the acoustic properties at the boundary of the QD [68], a QD placed close to a surface [69, in free-standing slab [69, 70, or in a quantum wire [71, or a pair of QDs at a given distance [72, 73].

The physics described by the model defined by Eqs. (1), (44), and (8) is most easily understood by first concentrating on two simple limiting cases: (i) vanishing exciton-phonon coupling, i.e., $g_{\mathbf{q}}=0$, and (ii) no optical driving, i.e., $M=0$. In the first case, the dynamics resulting from the remaining model reduces to the well-known optical Bloch equations for electronic two- or four-level systems without relaxation [74. In general, the laser driving leads to a coherent superposition of the discrete dot states, which typically implies for the corresponding occupations an oscillatory behavior known as Rabi oscillations. The frequency of these oscillations depends on the characteristics of the pulse. For the simplest case of a TLS driven by a monochromatic laser field with constant amplitude $f$ the Rabi frequency $\Omega_{\text {Rabi }}$ is given by [74]:

$$
\Omega_{\mathrm{Rabi}}=\sqrt{\Delta^{2}+|f|^{2}} \text {. }
$$


In the dressed state picture these oscillations correspond to a coherent superposition of the two dressed states resulting in quantum beats oscillating with the difference frequency $\Omega_{+}-\Omega_{-}=\Omega_{\text {Rabi }}$. Most important for the discussion of this review, exciton or biexciton states are reached by the coherent Bloch-type dynamics as pure states only for special excitation conditions. For example, in the case of a TLS the exciton can only be prepared as a pure state for resonant excitation, i.e., for $\Delta=0$ and distinguished values of the pulse area $\theta$, that equal odd multiples of $\pi$. Here, the pulse area is given by:

$$
\theta=\int_{-\infty}^{\infty}|f(t)| d t
$$

The influence of phonons on the state preparation using resonant Rabi oscillations is summarized in Sec. 3.1.

In the second limiting case the model reduces to the so called independent Boson model which is known to be analytically solvable [75, 76, 77, 48. As the independent boson model represents a pure dephasing mechanism, i.e., there is no coupling between different electronic states, the electronic occupations stay constant in the course of time. However, the carrier-phonon coupling in the independent boson model mixes electronic and phononic degrees of freedom in the excited states and thus gives rise to a polaronic character to the eigenstates of the system.

When both, the carrier-light and the carrier-phonon coupling are present, the interplay of both interaction mechanisms leads to a variety of dynamical features not found in the limiting cases, such as phonon-assisted optical generation processes or phonon-induced modifications of Rabi oscillations, as will be discussed in detail in Sec. 3. Other interaction mechanisms than the coupling to acoustic phonons typically lead to relaxation channels that, at least at sufficiently low temperatures, act on longer time scales than those considered here for preparation purposes. As an example, we mention the radiative decay, that takes place on a time scale of hundreds of picoseconds up to nanoseconds [78, 79, 80].

For some applications, it is favorable to place the QD in a microcavity [81, 82 , 83, 84, 85, 86, where the dot interacts with confined photon modes. Also for dots in cavities phonons can play a decisive role, e.g., for the dephasing [87, 88, 89, 90, 91, the photon statistics [92, 58, 93, 94, the indistinguishability of photons 95] as well as for providing a dot-cavity coupling in the case of non-resonant QD and cavity modes where phenomena like off-resonant cavity feeding have been found [96, 82, 97, 98, 99, 100, 101, 102. Although such systems are not at the focus of the present review, we note in passing that in this case, additional relaxation mechanisms, in particular cavity losses, may become of importance.

For more elaborate applications in quantum information technology the question of upscaling arises. This means that systems consisting of more than a single QD have to be considered. Like in the case of a QD in a microcavity, also here new phonon-related aspects come into play. A detailed discussion of such systems is beyond the scope of this review. However, let us briefly discuss some of these new aspects. The next step in the upscaling is an extension to double QD systems. If the QDs are sufficiently close to each other a tunnel coupling between the electron and/or hole states of the individual QDs may arise leading to the appearance of delocalized states. Because of the similarity of this states with bonding or antibonding states these structures are also named QD molecules. This coupling, which can be controlled to a large degree by an external electric field, has been the subject of a large 
amount of work over the past years investigating both excitonic [103, 104, 105] and biexcitonic transitions [106, 107. In particular close to a resonance between spatially direct and indirect exciton states phonon-induced transitions can be strongly enhanced 108, 73, 109, 110, which can also be interpreted as phonon-assisted tunneling 111, 112. The formation of a molecular polaron can give rise to a phonon-induced optical transparency [113]. Even if the distance between the QDs is larger such that tunneling processes are negligible, the QDs can still be coupled via the Coulomb interaction which leads to an excitation transfer, often referred to as Förster coupling. Like the tunneling, this transfer is also affected by phonons [72]. Phonon wave packets emitted from one QD can modulate the optical response of a second QD [114. Phonons in general also give rise to the decay of entanglement between excitons in two QDs 115, which is an important aspect in quantum computation.

\subsection{Theoretical Methods}

Apart from its relevance for the laser-driven QD dynamics, the model defined by Eqs. (1), (4), and (8) is a prototype of a quantum dissipative system [116, 65, 117] and thus also serves as a test ground for different theoretical methods. It is therefore not surprising that a wealth of theoretical approaches has been actively used for exploring the resulting dynamics. Despite of the simplicity of the electronic structure, the model in fact represents a genuine many-particle system due to the coupling to a continuum of acoustic phonon modes and an unlimited occupation number of each mode. For an arbitrary laser driving no analytical solution of the model is known.

Only in the limiting case of ultrashort excitations exact analytical solutions can be obtained, because in this limit the influence of the phonons on the excitonic system during the presence of the pulse is negligible. This is a result of the high-frequency cut-off in the phonon spectral density [Eq. [10]], which leads to a decoupling at times $t \lesssim \omega_{c}^{-1}$. Closed form results for an arbitrary sequence of ultrashort excitations have been derived using a generating functions formalism [118, 119]. For preparation purposes, on the one hand, a short preparation time is desired. However, ultrashort excitations strongly restrict the available preparation schemes such that essentially only the traditional Rabi flopping strategy, as discussed in detail in Sec. 3.1. can be realized. Other schemes that turn out to be advantageous for certain purposes (cf. Secs. 3.2 and 3.3) require longer pulse durations. For such excitations, one has to rely on numerical methods. Most often, these methods fully account for the dotlight coupling but treat the exciton-phonon interaction within further approximations. Examples of such approaches are the time-convolutionless approach [116, 120, 121, the correlation expansion [122, 123, 124, 125, 126], different types of master equations [16, 53, 64, 127, 88, 128, 93] or time-dependent perturbation theory [129, 125]. For the Hamiltonian dynamics within the model established by Eqs. (11), (41) and (8), also a numerically complete treatment without any further approximations to the light-matter or the carrier-phonon coupling has been implemented using a real-time path-integral approach [130, 131, 132, 133, 134. Taking these numerically complete solutions as a benchmark, direct comparisons have been performed for the correlation expansion [135, 136, the time-convolutionless formulation [127, and master equation 127. approaches. These comparisons revealed that for parameters typical for state preparation protocols, i.e., sufficiently low temperatures, weak carrier-phonon couplings and not too long times, the correlation expansion, the time-convolutionless formulation as well as advanced master equation approaches essentially give the same 
results. A direct comparison of the correlation expansion and perturbation theory revealed that the latter fails much earlier than the other approaches [125], which is of course not unexpected.

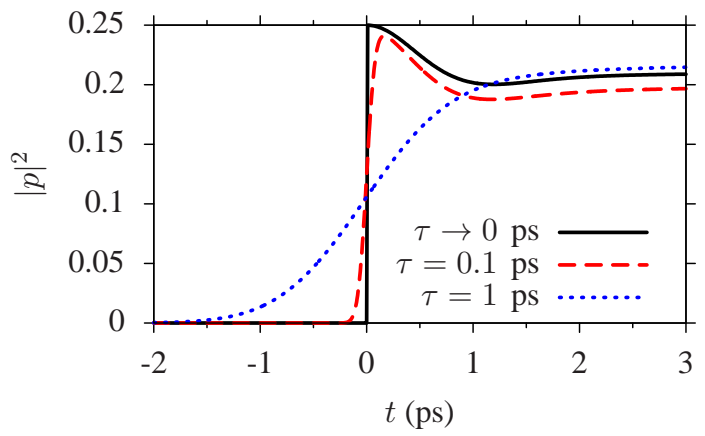

Figure 3. Modulus square of the polarization as a function of time after resonant excitation of a QD with an ultrashort pulse, a $100 \mathrm{fs}$ pulse and a $1 \mathrm{ps}$ pulse. All pulses have a pulse area $\theta=\pi / 2$.

A characteristic feature of the model defined by Eqs. (11), (4), and (8) is the fact that in the basis of the electronic states $|\nu\rangle$ the phonons only exhibit a diagonal coupling, i.e., they do not give rise to transitions between different states. Thus, after an optical excitation - when the light pulse has gone - the occupations of the states remain constant. However, the optical polarization, describing the coherence between the optically coupled states, exhibits an initial decay on a picosecond time scale. This reflects the pure dephasing nature of the model. As an example, the black solid line in Fig. 3 displays the time evolution of the modulus square of the optical polarization of a two-level dot generated by an ultrafast laser pulse with a pulse area $\pi / 2$. Like in an ideal TLS, an initial value of 0.25 is reached followed by a decay which is not only non-exponential but also only partial, i.e., the polarization approaches a temperature-dependent finite value at long times. The origin of this behavior as well as the dependence on the duration of the exciting pulse will be discussed in more detail in Sec. 3.1.3. Obviously, such a non-exponential and incomplete decay of the polarization, which in the spectral domain corresponds to strongly non-Lorentzian line shapes of the absorption or luminescence spectra [51, 50, 137, cannot be captured by a simple decay rate for the polarization. In fact, a naive calculation of phonon-induced relaxation rates according to Fermi's golden rule would give a zero result in this case, because the energies of the initial and final electronic state are the same and the phonon spectral density $J(\omega)$ is zero at $\omega=0$. Thus, the polarization decay represents a genuine non-Markovian dynamics. We note in passing, that the non-exponential decay towards a finite value is special for the superohmic coupling. In fact, in the subohmic case an exponential decay is found even when $J(\omega)$ still approaches zero for $\omega \rightarrow 0$ [65.

In view of these features, it may at first seem surprising, that even rather simple Markovian master equation approaches can, under certain circumstances, well reproduce experimental observations such as the damping of Rabi oscillations. Here, it should be noted that provided the laser envelope is slowly varying in time and the carrier-phonon coupling is not too strong, the dressed states become stable quasiparticles. As the light-matter interaction is already accounted for in the 
definition of the dressed states, the energy of the incoming photons is included in the corresponding energies. Therefore, the splitting between the dressed state energies is determined by the Rabi frequency which, for near resonant excitation is typically of the order of a few $\mathrm{meV}$, i.e., of the order of typical acoustic phonon energies. Therefore, the phonon coupling can now induce real transitions between the dressed states which can be described by a transition rate. Consequently, for moderate carrier phonon coupling strengths the phonons essentially lead to a thermalization dynamics in the dressed state basis [135] which is often well represented by a Markovian rate equation. A more detailed analysis based on master equations accounting for the phonon-induced memory reveals that the simple Markovian treatment comes to its limit, e.g., when phonon-induced renormalizations of the Rabi frequency become important: for stronger carrier-phonon coupling the stable quasiparticles assume a polaronic character, which can be used to formulate more advanced master equation approaches that rely on polaron transformations [64, 127, 88].

\section{State preparation schemes}

The state preparation of a QD typically aims at exciting the QD from its ground state, i.e., when no exciton is present, into a specific final state, e.g., a single exciton state, the biexciton state or a well-defined superposition state. In this section we will discuss three basically different optical control schemes and focus on the influence of the phonons on the state preparation. The most common excitation scheme relies on the coherent manipulation of the few-level system by resonant laser pulses. The pulses induce Rabi oscillations in the carrier system and drive the QD into a final state, which strongly depends on the pulse and material parameters such as pulse shape, pulse duration and dipole coupling matrix elements. The second scheme uses chirped pulses where the frequency sweeps over the resonance frequency of the QD starting either below or above the resonance. Here the excitation mechanism is an adiabatic rapid passage (ARP) from the ground state to the exciton or biexciton state which, as soon as the adiabaticity condition is reached, only weakly depends on the exact pulse parameters like the pulse area or the chirp rate. Both these schemes have in common that they work perfectly in the case of an isolated few-level system. Phonons deteriorate the controllability and therefore the study of the influence of phonons is mainly directed towards finding parameter ranges where this influence is of minor importance. In contrast to these two coherent schemes, the third excitation scheme is based on incoherent phonon-induced transitions to prepare the exciton or biexciton state by an excitation with a detuned laser pulse. This scheme therefore relies on the carrier-phonon interaction and works even better for stronger couplings. In the present section we will describe these schemes and summarize the experimental and theoretical work that has been performed in these fields.

\subsection{Resonant excitation}

When a QD modeled as an ideal TLS is excited resonantly, i.e., by monochromatic light with the frequency $\omega_{L}=\omega_{X}$, the occupations of the two states, here the ground and the exciton state, oscillate between zero and one as a function of time. These are the well-known Rabi oscillations, which were first described for spin oscillations in a rotating magnetic field [138. Without any further couplings the oscillation continues without any damping as long as the light field is present. A different but completely 
equivalent picture of Rabi oscillations is provided in the framework of the dressed state basis introduced in Sec. 2. Here the exciting light field gives rise to the splitting of the dressed states by the Rabi frequency and Rabi oscillations correspond to a quantum beat of these two states. The coupling to phonons disturbs these ideal Rabi oscillations. It is instructive to first discuss the influence of the phonons on Rabi oscillations in the time regime to understand their influence on the state preparation.

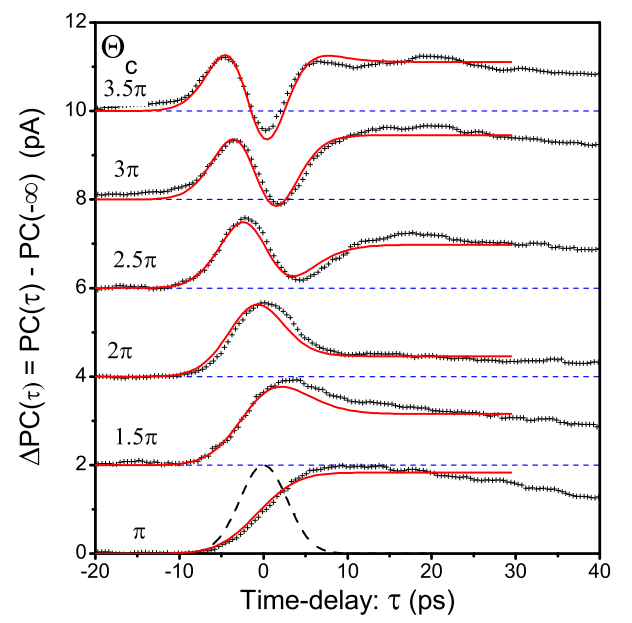

Figure 4. Experimentally measured Rabi oscillations as a function of time for increasing laser power. The dashed line marks the envelope of the laser pulse. The measurement has been performed by photocurrent detection of the time-dependent Autler-Townes-splitting from the exciton-to-biexciton transition. Figure reprinted with permission from Boyle et al [139].

3.1.1. Rabi oscillations The measurement of time-resolved Rabi oscillations for a single QD is experimentally challenging. To measure the time dependence of the Rabi oscillations, the change in the Autler-Townes-splitting in the TLS formed by the exciton-biexciton transition has been detected [139. The results are shown in Fig. 4 The Autler-Townes splitting refers to the splitting of the transition line caused by the coupling to the light field, when observed by a third (auxiliary) state [25]. The measurement has been performed using photocurrent detection [23] with an

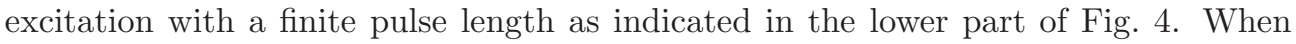
the laser power is increased, the Rabi frequency increases and more Rabi oscillations can take place during the pulse. Furthermore, the Rabi oscillations are damped and the damping increases with increasing power. Time-resolved Rabi oscillations have also been observed experimentally for a QD placed in a microcavity where the lightmatter interaction was in the strong coupling regime. Here, the Rabi oscillations reveal themselves in the time-resolved correlation function [140, 141, 142. Recently time-resolved Rabi oscillations have been measured by monitoring the time-dependent resonance fluorescence from a singly charged QD, where a TLS is formed by the negatively charged ground state and the trion state [143].

The coupling to phonons has several effects on the Rabi oscillations. First, it 
leads to a damping of the oscillations. The origin of this damping is most easily understood in the dressed state picture. In the case of Rabi oscillations the electronic system is in a superposition of the two dressed states. Phonons give rise to incoherent transitions between the dressed states. At low temperatures, when essentially only phonon emission processes are present, this leads to a relaxation to the lower dressed state, while at higher temperatures, when emission and absorption processes are almost balanced, both dressed states will be equally populated [135. In both cases the coherence between the states, which is responsible for the oscillations, decays.

In addition to the damping there are other phonon-induced phenomena. Due to the coupling to phonons, the excitation of the QD leads to a local lattice distortion in the region of the QD caused by the change of the electronic charge distribution. The resulting exciton-phonon complex is known as the polaron. The buildup of the polaron leads to a shift of the transition line from the bare value $\omega_{X}$ to the polaron-shifted value $\omega_{X}-\sum_{\mathbf{q}} \frac{\left|g_{\mathbf{q}}\right|^{2}}{\omega_{\mathbf{q}}}$ [51]. Accordingly, when modeling resonant excitations based on the Hamiltonian in Eqs. (1), (4) and (8), this shift has to be taken into account by adjusting the light frequency $\omega_{L}$ to the polaron-shifted transition frequency. The shift of the exciton line caused by the exciton-phonon interaction can be directly seen in experiments measuring either the passage of a strain pulse through the dot [144, 145] or by applying a periodic strain modulation in terms of a surface acoustic wave, where it leads to sidebands in the QD fluorescence spectrum [146]. It can be even exploited for an ultrafast switching of a QD placed in a microcavity into the lasing regime by shifting the transition frequency into resonance with the cavity mode 83 .

Finally, the polaronic nature does not only modify the energies of the states but also the coupling to the light field. This renormalization of the exciton-light interaction strength gives rise to a change in the frequency of the Rabi oscillations compared to its phonon-free value. This leads in particular to a temperature dependence of the Rabi frequency even if the light field is kept constant [129, 125].

An example of the dynamics of the two-level QD coupled to phonons is shown in Fig. . 5 , where the exciton occupation and the interband polarization, i.e., the coherence between ground and exciton state are displayed for the case of driving by a resonant light field with constant amplitude. Note that the polarization is displayed in a frame rotating with the light field. The Figure clearly shows the damping of the oscillations and the approach of a stationary state on a time scale of several tens of picoseconds. Both the damping rate and the oscillation frequency are temperature dependent, as discussed above. In the stationary state the occupation of the exciton level is 0.5 independent of the temperature, and the imaginary part of the polarization vanishes. The stationary value of the real part of the polarization, on the other hand, depends on the temperature. It has been found that the stationary state can be very well understood as a thermal distribution over the dressed states, confirming again the usefulness of this picture. Only for very low temperatures slight deviations have been found [135]. Here, both path integral and correlation expansion calculations revealed a stationary value of the polarization lying between the predictions by a weak coupling theory [65] and the thermal distribution over the dressed states.

For light, which is not strictly circularly polarized, in general both exciton states and also the biexciton are excited. Typically the exciton-to-biexciton transition energy lies below the ground state-to-exciton transition energy by a few meV, the biexciton binding energy $\Delta_{B}$ 147, 23, 148, such that a two-photon process can be chosen which is resonant on the ground state-to-biexciton transition but where the single-photon 


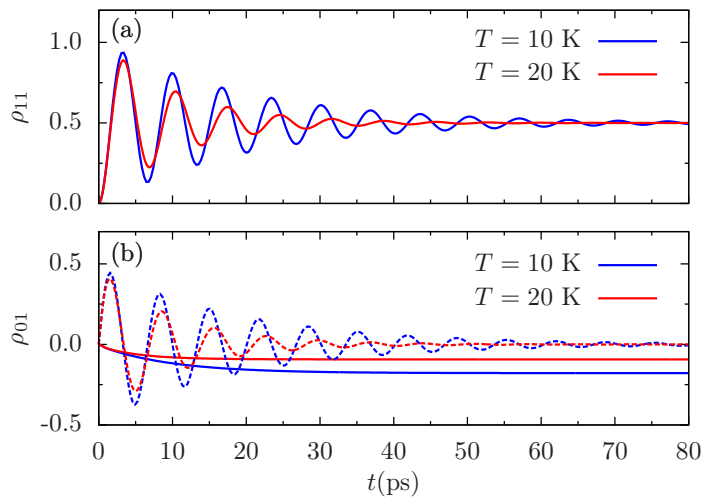

Figure 5. Dynamics of the TLS coupled to phonons for the case of resonant excitation by a constant light field switched on at $t=0$. (a) Occupation of the exciton state $\rho_{11}$ and (b) real (solid) and imaginary (dashed) part of the coherence $\rho_{01}$ in a frame rotating with the light field. Blue and red curves correspond to the temperatures $10 \mathrm{~K}$ and $20 \mathrm{~K}$, respectively. Figure reprinted with permission from Glässl et al [135].

frequency is sufficiently detuned from the ground state-to-exciton and exciton-tobiexciton frequencies. This leads to two-photon Rabi oscillations between the ground and biexciton state [149, 150, 18, 151. The impact of phonons is similar as in the two-level case 120 . The stationary values of the occupation and also the time scale on which the stationary state is reached, depend strongly on the polarization of the exciting laser pulses [134, 47.

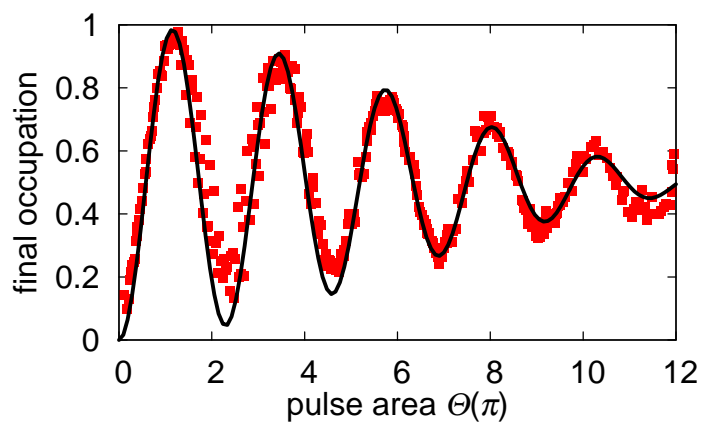

Figure 6. Occupation of the exciton state as a function of the pulse area at $T=5 \mathrm{~K}$ obtained from photocurrent measurements (dots) and calculated using the model discussed in the paper (solid line). We thank A. J. Ramsay for providing us with the experimental data originally published in 54.

3.1.2. Rabi rotations For an excitation of the $\mathrm{QD}$ with laser pulses the dynamics of the occupations are restricted to the time when the light field couples to the QD. After the pulse the occupations remain constant as long as radiative decay can be disregarded. Therefore it is meaningful to look at the final occupation after such an excitation. This is indeed the typical type of experiment to monitor the Rabi-type dynamics of excitons and biexcitons. Typically the final occupation is plotted as a function of the pulse area, which for a fixed pulse duration is proportional to the 
square root of the light intensity. Also here an oscillatory behavior shows up, which is commonly named Rabi rotation because in a TLS it reflects the rotation of the Bloch vector into a new direction by the action of the pulse. An example of both measured and calculated Rabi rotations is shown in Fig. 6, In an ideal TLS pulse areas given by odd multiples of $\pi$ result in a complete excitation of the system (occupation of one) while for even multiples of $\pi$ the system is completely driven back into the ground state. Rabi rotations are a widely used tool for state preparation, because they allow a preparation of pure exciton or biexciton states as well as of coherent superpositions with arbitrary amplitudes of ground, exciton and biexciton state to be carried out. The price which has to be paid for this versatility is the very sensitive dependence of the dynamics and thus of the generated state on the pulse parameters such as pulse shape, intensity and duration, as well as on material parameters like the energies or dipole coupling matrix elements. Already small deviations from the resonance or from the correct pulse area have a large influence on the state preparation. Therefore, for an ensemble of QDs, which typically have a range of transition frequencies and dipole matrix elements, a controlled state preparation using Rabi rotations is not possible.

First experiments showing Rabi rotations in a single QD, being the prototype of a coherent manipulation of a $\mathrm{QD}$, have been realized more than ten years ago in different structures [152, 153, 154, 155, 156. Since then, many other experiments have been performed and nowadays Rabi rotations are commonly used in many optical control experiments [157, 158, 159, 160, 161, 162, 54, 53, 148, 163, 164.

The coupling of the QD to its environment leads to a temporal damping of Rabi oscillations, as discussed above. This damping is reflected in a corresponding damping of Rabi rotations when plotted as a function of the pulse area, which has been investigated in a variety of experimental studies. Depending on the sample structure and the excitation conditions different coupling mechanisms may be responsible for the observed damping. For example, the intensity-dependent damping of Rabi rotations with a pulse area up to $10 \pi$ has been attributed to simultaneous bound-to-continuum transitions involving wetting layer states [158. A very good agreement of the damping behavior of measured Rabi rotations with pulse areas up to $12 \pi$ and theoretical calculations including the electron-phonon coupling as described in Sec. 2 has been recently demonstrated [53, 54 (see also Fig. 6). The calculations were able to reproduce the experimental data for a large temperature range from $5 \mathrm{~K}$ up to $75 \mathrm{~K}$. Also for QDs embedded in a one-dimensional wave guide the main source for the damping of Rabi rotations has been attributed to the coupling to acoustic phonons with additional contributions resulting from the enhanced radiative decay due to the strong coupling between the QD and the optical wave guide mode [164.

The experimental realization of Rabi rotations between the biexciton state and an exciton state 165, as well as between the biexciton and the ground state 149, 150, 18, 151, 166, 167, which can be both described in the four-level model, has great potential impact on the field of quantum logic where exciton-biexciton systems have been proposed as two-qubit quantum gates [21, 165] or as emitters of entangled photon pairs [11, 12, 13, 14, 15, 16, 17, 18. Rabi rotations between the ground and the biexciton state were also preformed by using two-color excitations 168. A recent review of coherent optical control of QDs can be found in Ref. 25.

The impact of the interaction of a QD exciton with acoustic phonons on the damping of Rabi rotations has been the subject of many theoretical works 123, 129, 169, 125, 119, 120, 64, 127, 136, 170, 131, 171]. Besides analyzing specific experimental conditions, these studies have also been motivated by the exemplary 
character of the Hamiltonian describing a bosonic bath coupled to a two-level system 65, 48, 116. More general types of coupling to a bosonic bath have also been investigated [172, however, here we will restrict ourselves to the type of coupling introduced in Sec. 2.

Like for Rabi oscillations in the time domain, a renormalization of the frequency, here with respect to the pulse area, is found. However, while Rabi oscillations are always decaying in time, for Rabi rotations the damping has been found to be a nonmonotonic function of the pulse area. For small pulse areas the amplitude of the Rabi rotations decreases with increasing pulse area thus showing the expected damping behavior, while for high pulse areas the amplitude increases again, which resembles an undamping. However, it should be noted that this is not an undamping in the time regime such as, e.g., the revival of Rabi oscillations in the Jaynes-Cummings model [173. This behavior has therefore been termed the reappearance of Rabi rotations [131]; an example is shown in Fig. 7 .

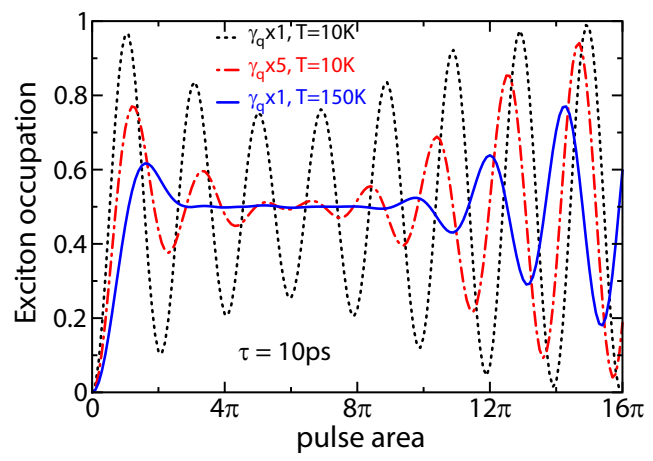

Figure 7. Exciton occupation as a function of the pulse area in the TLS calculated for the case of excitation by a 10 ps rectangular pulse at different temperatures and coupling strengths. Here, $\gamma_{\mathrm{q}}$ refers to the coupling $\left|g_{\mathrm{q}}\right|^{2}$ with the standard material GaAs parameters. For the dashed-dotted line this value has been increased by a factor of 5 . Figure reprinted with permission from Vagov et al 131.

The non-monotonic behavior of the damping can be traced back to the resonant nature of the exciton-phonon coupling matrix elements as reflected in the phonon spectral density given by Eq. (9) (see also Fig. 22). The spectral density clearly reveals that only phonons in a certain frequency window are efficiently coupled to the exciton and that there is a frequency $\omega_{\max }$ where the coupling is most efficient. As seen in Fig. 2. this frequency depends on the QD size; it can be roughly identified as the frequency of phonons with a wave length of the order of the QD size. The nonmonotonic behavior of the damping can now be understood in terms of a resonance between the exciton dynamics characterized by the Rabi frequency $\Omega_{\text {Rabi }}$ and the dynamics of the most strongly coupled phonons characterized by $\omega_{\max }$ [129, 125, 174. If these two frequencies coincide, the damping will be strongest. For much smaller Rabi frequencies $\left(\Omega_{\text {Rabi }} \ll \omega_{\max }\right)$ the electronic system oscillates so slowly that the phonon system follows essentially adiabatically and no energy is transferred irreversibly to the phonons. On the other hand, for Rabi frequencies much higher than the resonance 
$\left(\Omega_{\text {Rabi }} \gg \omega_{\max }\right)$ the change in the electronic system is so fast that the phonons cannot follow leading again to an inefficient coupling.

Instead of using the resonance argument in the time domain, also the spectral domain can be employed for the interpretation of the non-monotonic dependence of the damping on the Rabi frequency [125]. The driving light field gives rise to the appearance of dressed states with an energy splitting given by the Rabi frequency $\Omega_{\text {Rabi }}$. Phonon-induced transitions between the dressed states occur with a rate which is proportional to the phonon spectral density at the frequency corresponding to this energy splitting, which again explains why these transitions, and therefore the damping, are most pronounced when the Rabi frequency coincides with the position of the maximum of the spectral density, i.e., when $\Omega_{\mathrm{Rabi}} \approx \omega_{\max }$.

The non-monotonic damping and the reappearance of Rabi rotations are a general behavior related to the structure of the exciton-phonon coupling in QDs. As is seen in Fig. 7, it also occurs for a coupling which is much stronger than the one in typical QD structures and at rather high temperatures, when there are essentially no more oscillations visible in a certain window of Rabi frequencies. The details of the occurrence of the reappearance, however, strongly depend on many details of the exciting laser pulse, e.g., the pulse duration or the pulse shape. For pulses shorter than $\omega_{\max }^{-1}$ already for a $\pi$-pulse the excitonic dynamics is faster than the phonon dynamics such that already the first rotation is in the regime of decreasing damping. Therefore, Rabi rotations for short pulse excitations are essentially undamped [125]. The pulse shape determines the distribution of Rabi frequencies during the action of the pulse. A rectangular pulse is characterized by a single, fixed Rabi frequency during the pulse. Therefore there is a clear resonance and the reappearance sets in immediately above the resonance. In a Gaussian pulse, on the other hand, the Rabi frequency increases continuously from zero to a maximal value and then decreases again. Therefore, even if the amplitude at the pulse maximum is already beyond the resonance there are parts of the pulse in the leading and trailing edge where the amplitude satisfies the resonance condition and therefore a pronounced damping is present during these times. For this reason, in the case of a Gaussian pulse the amplitude of the Rabi rotations grows much slower after the onset of the reappearance and much higher pulse areas are required to reenter the regime of pronounced Rabi rotations [170.

Recent experiments on Rabi rotations with pulse areas up to $12 \pi$ have been quantitatively described by the model on which the present review is based [54, 53. Indeed for small pulse areas the influence of phonons is small and increases with increasing pulse area. The regime of reappearance of Rabi rotations has not yet been clearly reached in these experiments, even though a detailed comparison with calculations suggests that some features of the measurements can be regarded as first hints for an onset of the reappearance.

Both experimental and theoretical studies thus show that in order to achieve a high-fidelity state preparation based on a resonant excitation it is necessary to carefully select the excitation conditions regarding pulse length, pulse shape and pulse area. The optical control typically performs better when choosing short, rectangular pulses and working within a pulse area range up to $2 \pi$. Of course, when optimizing the excitation conditions the limitations of the model have to be kept in mind. For long pulses, other dephasing or relaxation mechanisms such as radiative decay may become effective [175]. Short pulses with their broad spectrum may excite other transitions either in the same dot or in nearby dots which also deteriorates the preparation of the desired state. 
Currently also the optical control of QDs in a microcavity has come into the focus of attention, where vacuum Rabi oscillations and the corresponding vacuum Rabi splitting are seen as indications for reaching the strong coupling regime between light and matter [176, 177, 178, 179. In the case of strong light-matter coupling there is a strong interplay between exciton-phonon and light-matter interaction, which for example can be seen in the line-width broadening in the Mollow-Triplet [180, 102]. The combined exciton-photon and exciton-phonon interaction in the strong coupling regime has been subject to many theoretical works $[181,58,87,90,95,88,89,128,182,91$. A detailed discussion of the phenomena related to the interplay between these coupling mechanisms is beyond the scope of this review.

3.1.3. Generation of superposition states So far we have mostly discussed the influence of phonons on the occupation of the QD states. For applications of optically controlled QDs in quantum information processing it is also necessary to prepare a given superposition state, e.g., a superposition of ground and exciton state [19]. One way to create a superposition state is to use a Rabi rotation with a pulse area, which is not equal to a multiple of $\pi$. For example, a $\pi / 2$ pulse applied to a TLS creates a superposition between ground and exciton state $(|G\rangle+i|X\rangle) / \sqrt{2}$. In the language of quantum information processing applying a $\pi / 2$ pulse corresponds to the action of a Hadamard gate on a qubit consisting of the states $|G\rangle$ and $|X\rangle[183$.

The ability to fully control the TLS is often demonstrated in Ramsey interference experiments, where a pair of two $\pi / 2$ pulses is used to resonantly excite the QD [154, 157, 184, 24, 25]. If the second pulse is in phase with the polarization created by the first pulse, there is constructive interference driving the system into the exciton state $|X\rangle$. If the second pulse has the opposite phase as the polarization there is destructive interference; the exciton is removed from the QD and the ground state $|G\rangle$ is restored. When plotted as a function of the phase difference between the pulses this leads in an ideal TLS to oscillations of the exciton occupation between zero and one. In the presence of dephasing processes these oscillations are damped.

Also for the preparation of superposition states the phonons are a major source of dephasing. Besides the dephasing during the action of the pulse, which can be reduced by using short pulses, the polarization is in general subject to dephasing even after the preparation of the state. This becomes clear for an excitation with ultrafast laser pulses, where the phonons cannot act during the pulse. After the generation of a superposition state the occupations remain constant, but the polarization decays as shown in Fig. [3 for the case of an excitation by pulses with a pulse area of $\pi / 2$ and three different pulse durations. In the case of ultrafast excitation the maximal polarization of 0.25 is reached. Directly after the generation a fast initial decay of the polarization within a time scale of about $1 \mathrm{ps}$ is found [51]. In the case of the $100 \mathrm{fs}$ pulse a slightly lower value is reached, but the behavior is still similar to the ultrafast limit. Because the ultrafast excitation of an exciton leads to an almost simultaneous change of the charge density, the equilibrium position of the crystal lattice changes. Due to the abrupt change, coherent phonons are created and in the case of acoustic phonons a coherent wave packet is sent out of the dot which carries away information from the QD [129, 185, 186, 174]. This leads to the observed dephasing of the coherence [51, 55]. After the wave packet has left the dot, the polarization exhibits no further decay caused by the phonons. The time scale of the initial decay is determined by the time the wave packet needs to leave the QD. Other interaction mechanisms, in particular radiative decay, give rise to an exponential decay on a much longer time scale 
78. The non-exponential decay of the polarization, which is a clear indication of the non-Markovian nature of the exciton-phonon coupling in the absence of a driving light field, has been measured using four-wave-mixing techniques [78, 187, 188, 189, 190].

From the polarization dynamics after an ultrafast excitation the absorption spectrum of the QD can be obtained, which consists of the zero phonon line and the typical phonon background resulting from the interaction with acoustic phonons. The interaction with optical phonons gives rise to satellites in the spectrum separated by multiples of the optical phonon frequency, which again are superimposed on a background due to the acoustic phonons. This type of spectra has been seen in many experimental and theoretical studies [50, 78, 51, 62, 67.

For longer pulses, the change of the electronic charge density caused by the excitation of the QD is much slower and, for sufficiently long pulses in the adiabatic limit no wave packet is emitted. Thus, in the polarization no decay of the polarization after the pulse is found, as shown in Fig. 3 for the case of an excitation by a 1 ps pulse. Instead, coherence is lost already during the action of the pulse, which also results in a reduced occupation of the states. Interestingly, the final polarization long after the pulse, say for $t>2 \mathrm{ps}$, is larger in the case of the longer pulse. Instead of using a single long pulse a sequence of ultrashort pulses can be used, which for suitable parameters also gives rise to a reduced decoherence compared to a single ultrashort pulse [191, 56].

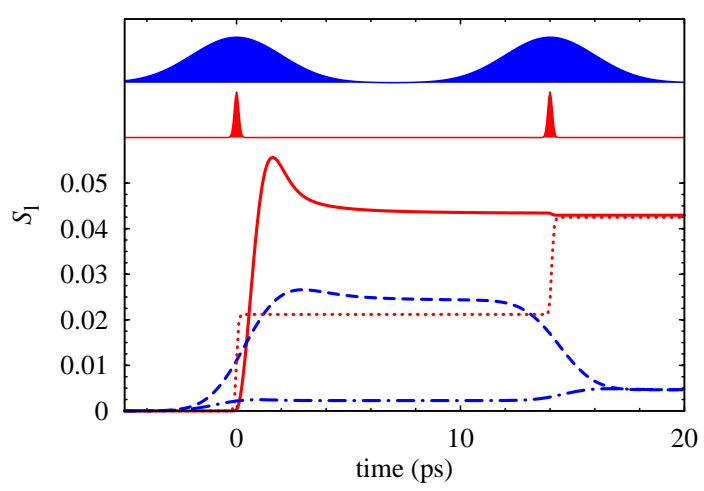

Figure 8. Linear entropy as a function of time for the case of excitation by two $\pi / 2$ pulses. Red (solid and dotted) curves: excitation by two 100 fs pulses, blue (dashed and dashed-dotted) curves: excitation by two 2 ps pulses. The solid and dashed lines refer to a calculation of the entropy for the bare qubit (ground and exciton state), the dotted and dashed-dotted lines are calculated for the dressed qubit (ground and polaron state). The pulse envelopes are shown in the upper part (in arbitrary units). Figure reprinted with permission from Machnikowski et al [192.

It is interesting to note that the reduced value of the polarization after the longer $\pi / 2$ pulse seen in Fig. 3 compared to the ideal value of 0.25 is not completely related to irreversibility, in contrast to the decay after an ultrafast excitation, where irreversibility results from the emission of the phonon wave packet. This is seen when comparing a Ramsey-type interference using short and long pulses 192 and calculating the linear entropy. The linear entropy $S_{1}=1-\ln \left(\rho^{2}\right)$, where $\rho$ is the reduced density matrix in the QD subspace, is a suitable measure for the purity of a QD state [116]. The results of such calculations are shown in Fig. 8 (solid and dashed 
lines). The first pulse drives the system into an entangled state between exciton and phonons and thus increases the linear entropy. While in the case of short pulses the linear entropy essentially remains constant after the second pulse, for long pulses it is considerably reduced by the second pulse. In fact, it turns out that in the case of an excitation by long pulses the appropriate definition of a qubit should include the polaron dressing of the exciton [192]. The dotted and dashed-dotted lines in Fig. 8 show the results for the linear entropy when the reduced density matrix is calculated in the polaron basis. In this case, the entropy after the first pulse is much smaller than in the bare exciton basis (solid and dashed lines), in particular for the long pulse, and the entropy never decreases. The dressed qubit therefore undergoes much lower dephasing and much higher fidelities of quantum gate operations can be achieved. However, when strongly increasing the pulse duration other dephasing mechanisms such as radiative decay usually become more important. Taking into account this trade-off between different types of decoherence then leads to optimal pulse durations which depend on the specific QD structure as well as on temperature [175].

\subsection{Excitation with chirped laser pulses}

A different method of state preparation is achieved by using chirped laser pulses. During these frequency-modulated pulses the frequency varies with time. In the most simple case the modulation is linear, such that for a pulse with pulse maximum at time $t=0$ the corresponding laser frequency is given by $\omega(t)=\omega_{0}+a t$, where $\omega_{0}$ is the central frequency of the laser pulse at the pulse maximum, and $a$ is the chirp rate. In most cases, $\omega_{0}$ is taken to be resonant with the exciton transition. For the generation of chirped laser pulses a transform-limited pulse is sent through a chirp filter characterized by a chirp coefficient $\alpha$ [193, 194. In such a filter an initial Gaussian pulse with amplitude $f_{0}$ and pulse duration $\tau_{0}$ is transformed into a chirped Gaussian pulse with pulse duration $\tau$, chirp rate $a$ and amplitude $\tilde{f}$, given by

$$
\tau=\tau_{0} \sqrt{1+\frac{\alpha^{2}}{\tau_{0}^{4}}}, \quad a=\frac{\alpha}{\alpha^{2}+\tau_{0}^{4}}, \quad \text { and } \quad \tilde{f}=f_{0} \sqrt{\frac{\tau_{0}}{\tau}},
$$

respectively [195].

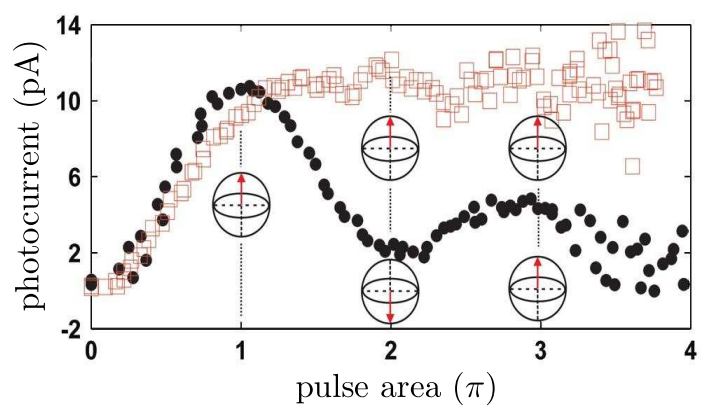

Figure 9. Exciton occupation as a function of the pulse area obtained from photocurrent measurements for the case of resonant excitation by a 2 ps transformlimited pulse (solid circles) and an excitation by a 15 ps chirped pulse (open squares). Figure reprinted with permission from Wu et al 196].

The use of chirped laser pulses in atomic systems is a well established tool [199, 200] and recently this technique has been transferred to single QDs, where a 


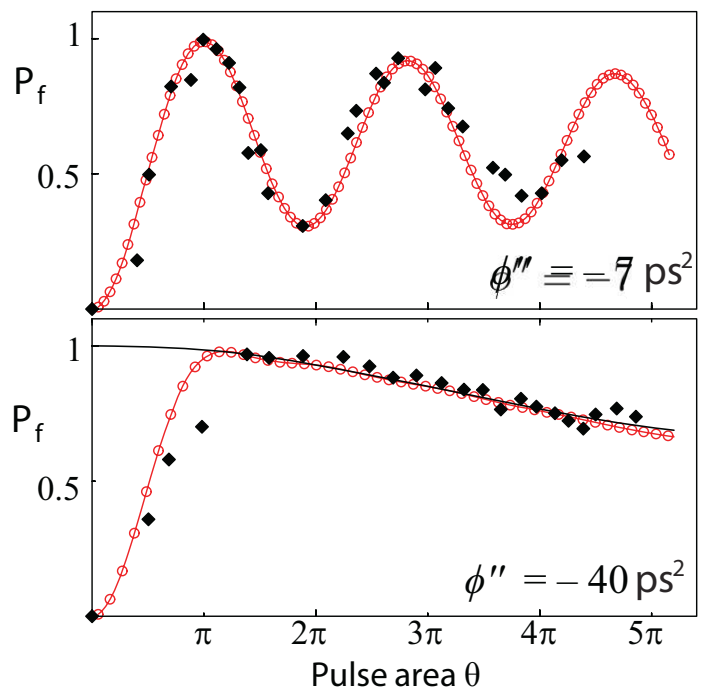

Figure 10. Exciton occupation $P_{f}$ as a function of the pulse area for a very small (upper panel) and a large negative chirp (lower panel). Diamonds show the experimental results from Ref. [197], circles are obtained from numerical simulations and the solid lines shows results from an analytical approximation valid in the adiabatic regime. Note that $\phi^{\prime \prime}$ is twice the chirp coefficient $\alpha$ defined in the text. Figure reprinted with permission from Debnath et al [198.

stable population inversion has been experimentally demonstrated using a linear chirp [196, 197] (see Fig. 9 and the experimental points in Fig. 10). The idea of population transfer via adiabatic rapid passage (ARP) in a TLS can be easily understood in the dressed state picture, as already introduced in Sec. 22 When the system evolves adiabatically, at each time step a diagonalization of the Hamiltonian can be performed leading to time-dependent dressed states. The dressed states for a two-level QD under excitation with a linearly chirped laser pulse with a duration of 5 ps are shown in Fig. 11] Before and after the pulse the dressed states can be identified with the system eigenstates, namely the ground state $|G\rangle$ and the exciton state $|X\rangle$. At the pulse maximum $(t=0)$ both dressed states are given by equal superpositions of $|G\rangle$ and $|X\rangle$. When following one of the branches, it changes its character continuously from the ground state $|G\rangle$ to the exciton state $|X\rangle$ or vice versa. The requirement for ARP, namely that the system follows the branch adiabatically, is quantified in the Landau-Zener criterion $|f|^{2} \gg|a|\left[200,201,202\right.$, and the condition $a \tau^{2} \gg 1$ [193]. Therefore, as soon as the adiabatic threshold is overcome, by following one of the branches a robust and complete population inversion of the system can be achieved. The robustness of ARP refers to the fact, that variations in the pulse area, the pulse duration and/or the chirp rate do not affect the state preparation, as long as the adiabaticity condition is not violated. Because of this insensitivity ARP has also been predicted for ensembles of QDs [203, provided the distribution of energies and dipole matrix elements of the QDs is such that the adiabaticity condition is fulfilled for the whole ensemble.

In an ideal TLS by using the ARP scheme, like in the case of resonant excitation, an exciton preparation with arbitrarily high fidelity can be achieved. However, also here phonons act as a limiting factor. Let us discuss the influence of the phonons 

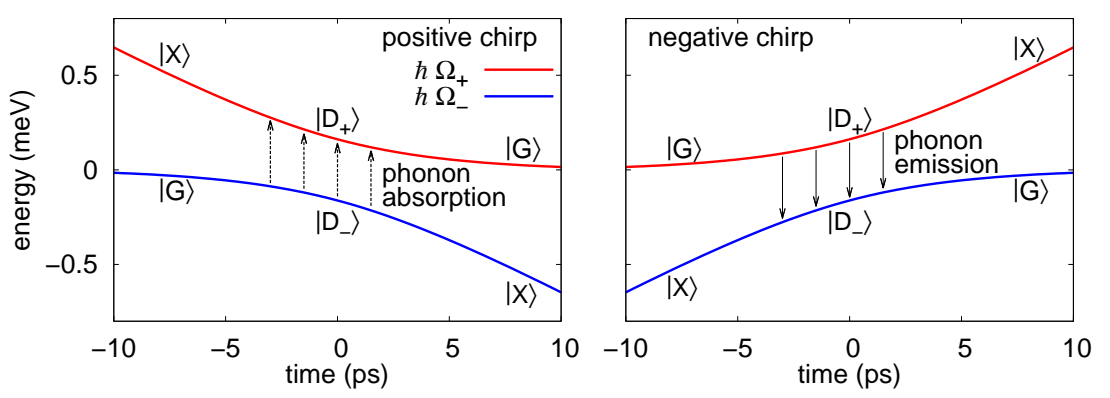

Figure 11. Schematic plot showing the time evolution of the dressed states in a two-level system excited by a chirped laser pulse for positive (left panel) and negative (right panel) chirp.

on the final occupation of the system after the action of the laser pulse as a function of the pulse area. The experimental data in the lower part of Fig. 10 show that the exciton state occupation approaches unity for pulse areas between $\pi$ and $2 \pi$ and then decreases with increasing pulse area. Such a decrease is absent in an ideal TLS and is attributed to phonons.

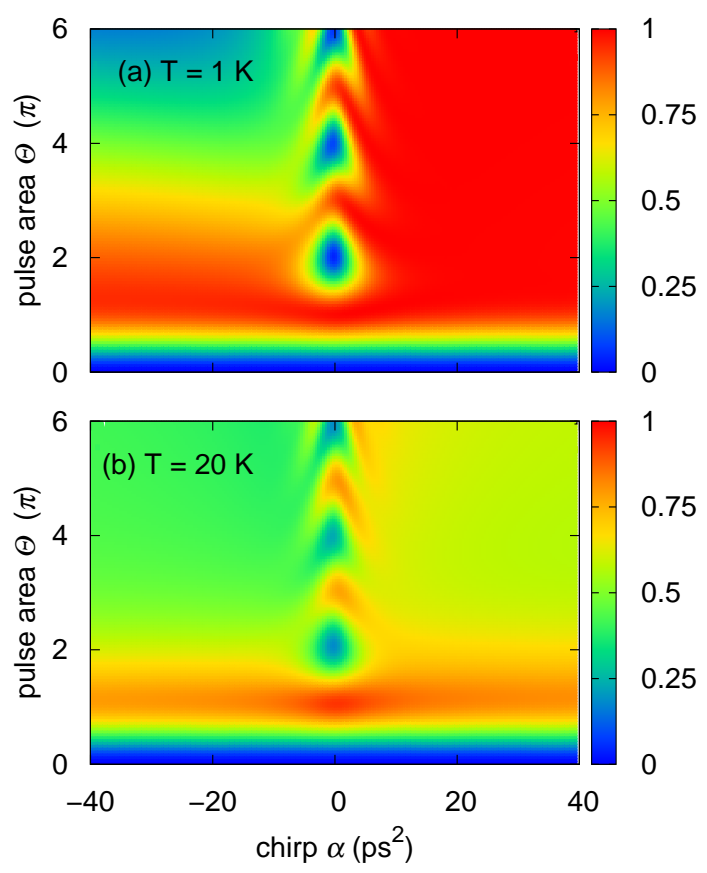

Figure 12. Calculated exciton occupation as a function of pulse area and chirp coefficient at two different temperatures. Figure reprinted with permission from Lüker et al 204].

Theoretical results for the final exciton occupation at two different temperatures and over a wide range of chirp coefficients and pulse areas are shown in Fig. 12, These calculations reveal, that for low temperatures and positive chirps the state preparation is almost not affected by phonons, while for negative chirps a high-fidelity exciton 
generation is restricted to pulse areas between about $\pi$ and $2 \pi$. For higher pulse areas the efficiency considerably drops even at low temperatures. This is in agreement with the experimental and theoretical results shown in Fig. 10. The influence of the phonons on the ARP process can be well understood in the dressed state picture. Here the phonons give rise to transitions between the adiabatic eigenstates. A transition from the upper to the lower branch occurs via the emission of a phonon, while the reverse process is associated with the absorption of a phonon. At low temperatures, when essentially no phonons are present, phonon absorption is highly unlikely resulting in a strong asymmetry between the paths. In the case of a positive chirp the system evolves along the lower branch and is therefore almost unaffected by phonons, while for a negative chirp the evolution is along the upper path and even at low temperatures phonon emission leads to pronounced transitions to the lower branch. This asymmetry has been found in all calculations based on different levels of complexity [204, 171, 198, 205] and has been seen in recent experiments [206. The interpretation is confirmed by an analytical model, where the phonon transition rates between the dressed states are calculated from the phonon spectral density [Eq. (9)] at the frequency corresponding to the instantaneous energy splitting of the dressed states 198. The results of this model are shown as the solid black line in the bottom panel of Fig. 10. As soon as the condition for adiabatic evolution is reached, i.e., for pulse areas above about $\pi$, the model is in perfect agreement with the full calculation and with the experimental results. For very high pulse areas calculations predict that the efficiency should increase again because, like in the case of the reappearance of Rabi rotations, the splitting between the dressed states becomes larger than the cut-off frequency $\omega_{c}$ in the phonon spectral density [171. With increasing temperatures the rate of phonon absorption approaches the phonon emission rate and thus the asymmetry between positive and negative chirp disappears 204, 198. The ARP scheme then becomes less efficient except for a pulse area in a small range around $\pi$ where phonon-induced transitions are not yet very efficient (see Fig. 12(b)).

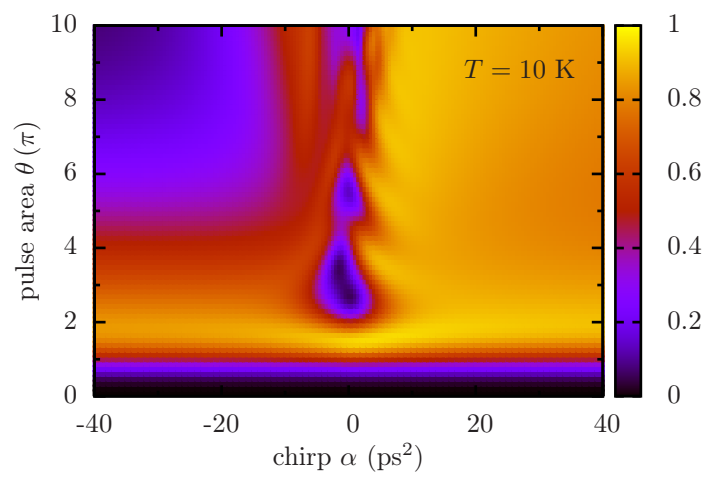

Figure 13. Calculated biexciton occupation as a function of pulse area and chirp coefficient for the case of a two-color excitation using circularly polarized pulses. The frequencies at the pulse maximum of the two pulses are taken to coincide with the ground state-to-exciton and the exciton-to-biexciton transition, respectively. Figure reprinted with permission from Glässl et al [207.

When exciting the QD with linearly polarized chirped pulses transitions to the biexciton state become possible. In this case, also for positive chirps the efficiency of exciton state preparation may be reduced due to a phonon-assisted generation of 
the biexciton, which becomes important for small biexciton binding energies [121. On the other hand, this also opens up the possibility of a direct preparation of the biexciton state using ARP, a process which is very attractive because of the high potential use of biexciton states in quantum information technology [208, 207, 209]. Two protocols either via direct two-photon absorption resonant on the ground stateto-biexciton transition or via two-color excitations have been proposed. In the first case, a linearly polarized pulse is used to directly excite the biexciton from the ground state. Because of the off-resonant coupling to the exciton state higher pulse areas are required to reach the adiabatic regime. As soon as this threshold is overcome, a stable biexciton generation via ARP is possible, which can again be understood in the dressed state picture [207. In the low temperature limit, the influence of phonons is asymmetric with respect to the sign of the chirp in the same way as for the exciton preparation. In the high temperature case, an overall damping is found, but also for the biexciton preparation it is possible to decouple the phonons from the electronic system by using pulses with a sufficiently high intensity such that the splitting between the dressed states becomes larger than the cut-off frequency for the phonon coupling. In this regime a robust and high-fidelity biexciton preparation using ARP has been predicted at temperatures as high as $80 \mathrm{~K}$ [209]. Instead of using a linearly polarized pulse the two-color excitation scheme is based on the excitation by a pair of chirped pulses with opposite circular polarization. The frequency at the pulse maximum of one of the pulses is tuned to the ground state-to-exciton and of the other to the exciton-tobiexciton transition. Figure 13 shows that this scheme again gives rise to an efficient biexciton generation. The drawback of using two different pulses is compensated by the fact that the adiabatic threshold lies at lower pulse areas compared to the twophoton scheme [207.

\subsection{Phonon assisted exciton and biexciton preparation}

A Rabi-type dynamics is not restricted to the case of resonant driving but can also be observed under off-resonant driving conditions. In an ideal TLS without dissipation, off-resonant Rabi dynamics is characterized by oscillations of small amplitude and high frequency [cf. Eq. (12)] and not sensitive to the sign of the detuning [74. This behavior is significantly changed by the interaction with phonons. As in the resonant case, the oscillations are damped and, for larger detunings, where in the phonon-free case the amplitude is small, they are hardly resolved [210, 135, 171, 211. In addition, at low temperatures there is a strong asymmetry between positive and negative detunings. For positive detunings, i.e., a laser frequency above the resonance, superimposed on the weak oscillations there is a growth of the exciton occupation as a function of time, whereas it decreases for negative detunings [135, 212. This asymmetry reflects a thermalization in the dressed state basis and can be traced back to the fact that immediately after switching on a pulse with positive detuning, the dressed state with the higher ground state contribution is more strongly occupied. For positive detunings this is the upper dressed state [cf. Eq. [6)] while for negative detunings it is the lower one. Thermalization at low temperatures is mainly governed by phonon emission processes leading at longer times to a dominant occupation of the lower dressed state, which for positive detunings has a large exciton state contribution. Thus, for positive detunings the exciton state occupation increases, while for negative detunings it remains small or even decreases. With increasing temperature phonon

absorption processes become more important leading, like in the case of chirped pulse 


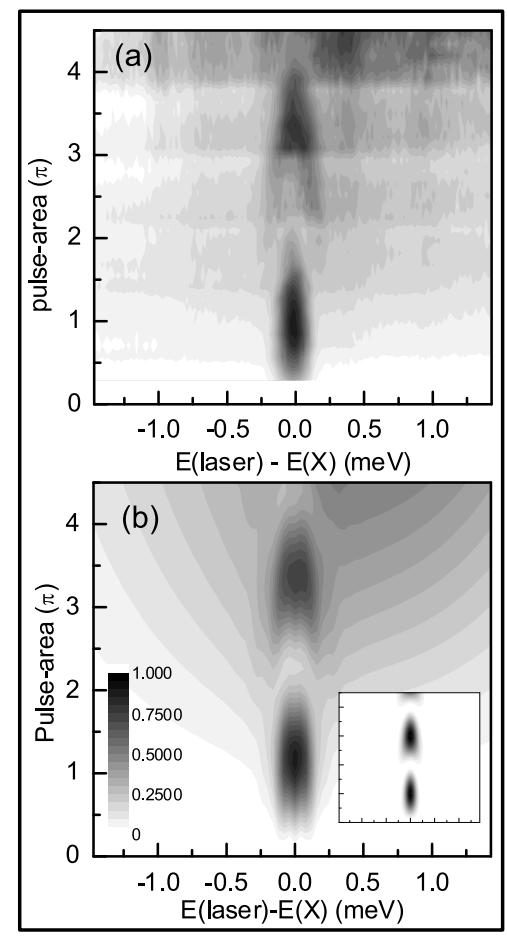

Figure 14. (a) Gray scale plot of the photocurrent of a single QD excited by a Gaussian pulse of 4 ps duration (full width at half maximum) at a temperature of $15 \mathrm{~K}$ measured as a function of laser detuning and pulse area. (b) Calculation of the photocurrent using a Markovian master equation approach. (Inset) Calculation without dephasing. Figure reprinted with permission from Ramsay et al 210.

excitations, finally to an equilibration between the dressed states and thus also between ground and exciton state.

This asymmetry has also been demonstrated in experiments using pulsed excitations [210]. Figure 14(a) shows a photocurrent spectrum of the QD occupation as a function of the detuning and the pulse area. Clearly seen are Rabi oscillations for near resonant excitations. The oscillation amplitude rapidly decays with rising detuning. The main signature of the phonon-induced asymmetry is an enhanced exciton occupation observed for positive detunings and higher pulse areas. Part (b) of Fig. 14 displays results of corresponding calculations based on a simple master equation approach that relies on Markovian rates for transitions between the dressed states. Obviously, all key features of the measurements are well reproduced on this level of the theory. Similar results have also been obtained by calculations within a correlation expansion for finite pulses [171. The calculations also reveal that the asymmetry originates from the phonon influences, as can be seen, e.g., by a comparison with simulations for the case without phonons shown in the inset of Fig. 14(b).

The interpretation in terms of a relaxation between the dressed states indicates that at low temperatures at sufficiently long times an essentially complete occupation of the lower dressed state should be achievable. Since for increasing positive detuning the lower dressed state becomes more and more exciton-like, there should be no 


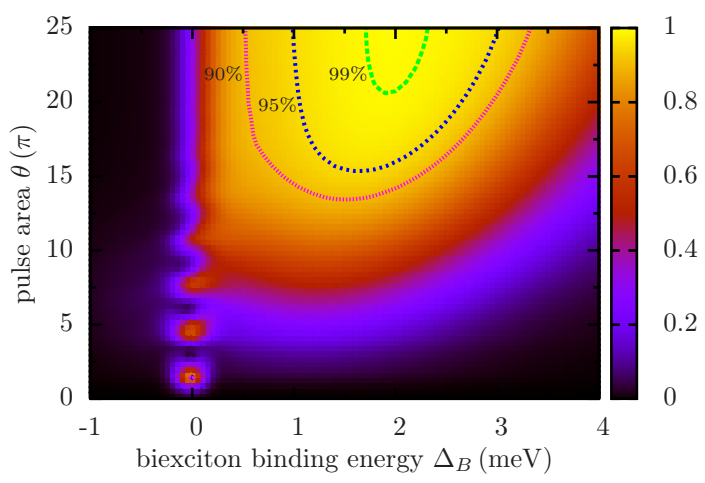

Figure 15. Final biexciton occupation after a Gaussian pulse with a FWHM of $15 \mathrm{ps}$ at $T=4 \mathrm{~K}$ as a function of the biexciton binding energy $\Delta_{B}$ and the pulse area $\theta$. The laser frequency is chosen in resonance with the ground state to exciton transition. The contour lines display where certain values of the biexciton occupation are reached. Figure reprinted with permission from Glässl et al 211 .

fundamental limitation in the final exciton state occupation. Indeed, calculations for excitations with a constant light field have shown that exciton occupations arbitrarily close to one can be reached [135]. However, in order to make off-resonant excitations useful for state preparation purposes, the process has also to be sufficiently fast such that other relaxation mechanisms not included in the model, e.g., the radiative decay, are not yet efficient in reducing the exciton occupation again. For dots without a cavity, this is not a very severe restriction, as the typical time scale for radiative decay is of the order of one nanosecond [78, 79, 80. For dots in cavities, often cavity losses are the main relaxation process [176, 213, which, depending on the quality factor, can demand for noticeably shorter preparation times.

A short time scale of phonon-induced relaxation and a high fidelity of exciton generation seem to be two conflicting ingredients. The phonon emission rate is determined on the one hand by the phonon spectral density [Eq. (9V] at the energy of the splitting between the dressed states, and on the other hand, since phonons only couple to the excitonic part of the electronic state, by the exciton contribution in the dressed states. This favors energy splittings in a certain energy window (cf. Fig. (2) and parameters such that both dressed states as given in Eq. (6) have a noticeable exciton contribution. To achieve a high fidelity, the lower dressed state that is eventually reached at the end of the preparation process, should be as close as possible to the exciton state. In the case of excitation by a rectangular pulse, when the character of the dressed states remains constant over the pulse, there is a conflict between these two requirements. The condition of a strongly exciton-dominated lower dressed state means that the upper dressed state almost coincides with the ground state giving rise to a very small overlap and thus to a very low phonon emission rate. The minimal time required to achieve a certain degree of fidelity is therefore determined by a tradeoff between these two opposite aspects. In the case of excitation by a Gaussian pulse the character of the dressed states changes over the duration of the pulse. Close to the pulse maximum a strong driving introduces a strong mixing between ground and exciton state in both dressed states, while for decreasing amplitude the lower dressed state evolves into the pure exciton state thus favoring a high-fidelity preparation. Analogous arguments given here for the exciton system hold for the exciton-biexciton 
system.

Recent calculations have shown that under realistic excitation conditions a highfidelity and robust phonon-assisted preparation targeted at either the exciton or the biexciton is indeed possible on a picosecond time scale 211. As an example, Fig. 15] displays results for the preparation of the biexciton where the final biexciton occupation after the pulse is plotted as a function of the biexciton binding energy $\Delta_{B}$ and the pulse area $\theta$ at $T=4 \mathrm{~K}$. In these calculations, a linearly polarized laser pulse with duration 15 ps FWHM has been tuned in resonance with the ground state-to-exciton transition. For vanishing $\Delta_{B}$, the excitation is also resonant to the exciton-to-biexciton transition, and two-photon Rabi oscillations between the ground and the biexciton state are observed. For finite $\Delta_{B}$, in turn, the exciton-to-biexciton transition is off-resonant and for positive $\Delta_{B}$, i.e., when the biexciton energy is lower than twice the exciton energy, the biexciton occupation increases reflecting the fact that the lowest dressed state, towards which the system relaxes due to the carrierphonon interaction, has a dominant biexciton contribution. It turns out that for a broad range of typical biexciton binding energies and sufficiently high pulse areas an almost perfect preparation with biexciton occupations close to one can be achieved. The phonon-coupling between the dressed states turns out to be sufficiently strong, such that the final values plotted in Fig. 15] which document a preparation of an almost pure biexciton state, are reached on a time scale of $\sim 10 \mathrm{ps}$ [211]. In order to realize such short preparation times, one exploits the fact that the phonon-induced relaxation strongly depends on the system parameters such as the QD size and the electron-phonon coupling strength as well as on the driving parameters such as the pulse area and the pulse length. Once the parameters are chosen in a regime favorable for fast relaxation, the protocol turns out to be robust with respect to changes in the pulse area as well as to the precise value of the biexciton binding energy.

Being caused by phonon emission processes, the state preparation speeds up, when the carrier-phonon coupling increases. Like all the other results discussed in this review, the calculations shown in Fig. 15 correspond to the weak coupling limit realized for InGaAs-type QDs, while for other materials such as, e.g., GaN, the carrierphonon coupling can be substantially stronger [55, 67]. Thus, in contrast to the other preparation protocols discussed in this review, which work best in the absence of the carrier-phonon coupling, the phonon-assisted preparation based on off-resonant driving performs the better the stronger the carrier-phonon coupling is 211. Finally, we note that the high biexciton occupations found in Fig. 15 also imply that the exciton state is eventually unoccupied even though it is resonantly driven. This is because the lowest dressed state has an almost vanishing exciton contribution. In fact, the resonant driving of the exciton with linearly polarized laser pulses turns out to be most favorable for preparing the biexciton. Using lasers that are off-resonant to all transitions in the exciton-biexciton system is typically less efficient.

Similar to the biexciton state preparation also the single exciton state can be efficiently populated by a phonon-assisted process 211. This is achieved by a circularly polarized excitation - in order to suppress the coupling to the biexciton - by a laser pulse which is positively detuned with respect to the ground state-toexciton transition. For such an excitation, Fig. 16(a) illustrates the dependence of the exciton occupation after the pulse on the detuning $\Delta$. For small and large $\Delta$, low exciton occupations are found, while high occupations are obtained for intermediate $\Delta$, reflecting the finite energy window of efficient carrier-phonon coupling in the phonon spectral density (cf. Fig. 22). In Fig. 16(b) the final exciton occupation is plotted as a 


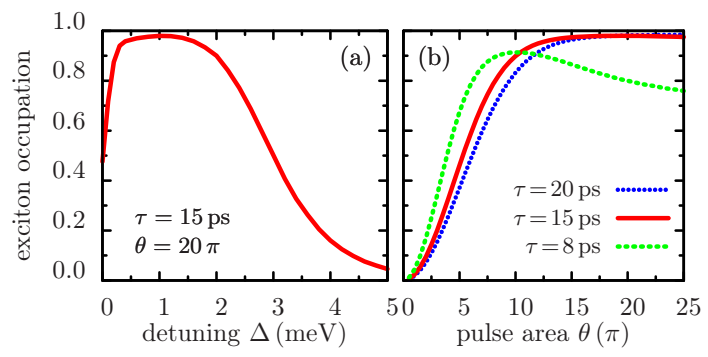

Figure 16. Final exciton occupation after a Gaussian pulse with pulse length $\tau$ (FWHM) at $T=4 \mathrm{~K}$, (a) as a function of $\Delta$ for $\tau=15 \mathrm{ps}$ and $\theta=20 \pi$, and (b) as a function of $\theta$ for $\Delta=1 \mathrm{meV}$ and different values of $\tau$ as indicated. Figure reprinted with permission from Glässl et al [211].

function of the pulse area for several pulse lengths. Clearly, when the pulse length is below $10 \mathrm{ps}$, the maximal occupation that can be reached is noticeably lower than one and quickly further degrades when the pulse area is not optimally chosen. However, already for pulse durations above $15 \mathrm{ps}$, the final exciton occupation stays close to one for a broad range of pulse areas. Figure 16 therefore again confirms the robustness of the phonon-assisted scheme against variations of detuning, pulse area and pulse length within certain windows determined by material and excitation parameters.

\section{Conclusions}

In this review we have discussed various schemes that have been used for the preparation of excitonic and biexcitonic states in self-assembled QDs by means of optical excitations. The focus of the discussion was on the role of phonons for these excitation schemes. Three basically different schemes have been presented in the previous section: (i) an excitation by resonant laser pulses employing Rabi oscillations, (ii) an excitation by using chirped laser pulses based on ARP, and (iii) an excitation by detuned laser pulses relying on phonon-assisted exciton or biexciton generation. In the following we will compare these schemes regarding different aspects and discuss the advantages, drawbacks, and limitations of the respective scheme.

First of all, when talking about state generation one has to distinguish between the generation of pure exciton or biexciton states and the generation of arbitrary superposition states. Of the three schemes discussed in this review only the resonant excitation can be used for the preparation of superposition states. Superposition states are in general subject to phonon-induced dephasing after their generation. However, due to the non-Markovian nature of the pure dephasing process this dephasing is not complete. By excitation with not too short laser pulses the irreversibility which is associated with the emission of a phonon wave packet can be reduced. For long pulses, on the other hand, the dephasing during the excitation process caused by phonon-induced transitions between the dressed states as well as by other dephasing mechanisms becomes more important, such that an optimum for the pulse duration exists. The fidelity of the state preparation in this case strongly depends on the details of the QD structure and on the temperature.

Pure exciton and biexciton states can be prepared by all three mechanisms. For all three schemes there is no fundamental limitation of the achievable fidelity caused by the interaction with phonons, although limitations may arise from the approximations 
inherent in the model, e.g., by neglecting higher excitonic states as well as other coupling mechanisms, in particular the radiative decay. In the case of Rabi rotations the fidelity of exciton or biexciton preparation can be made arbitrarily high in the limit of ultrafast excitations, because here the dynamics in the exciton system becomes much faster than the phonon dynamics such that the phonons cannot follow. In the ARP scheme using excitations with chirped pulses a high-fidelity generation of excitons and biexcitons can be reached. In the case of positive chirps at sufficiently low temperatures, when phonon absorption processes are negligible, a high-fidelity preparation is possible in a wide range of pulse areas while for negative chirps the pulse areas are restricted to a window between about $\pi$ and $2 \pi$. For excitations with detuned pulses exciton and biexciton occupations arbitrarily close to one can be achieved by using sufficiently long pulses at low temperatures such that the relaxation from the upper to the lower dressed state by phonon emission is essentially complete and phonon absorption processes can be neglected.

A clear advantage of schemes (ii) and (iii) over the resonant excitation scheme (i) is their robustness with respect to pulse and system parameters. Once a certain threshold of pulse intensity and pulse duration is overcome, the exciton or biexciton generation is rather insensitive to variations of these parameters. This has the advantage that no detailed knowledge of the individual QD is required to perform the state preparation. Furthermore, for this reason the schemes also work for ensembles of QDs with a distribution of energies and dipole matrix elements, as long as these distributions are sufficiently narrow. In the case of ARP the adiabaticity condition has to be fulfilled for the whole ensemble, in the case of off-resonant excitation the splitting of the dressed states of each QD in the ensemble has to be in the range such that phonon emission occurs with a sufficient rate and phonon absorption processes are negligible.

When comparing the robust schemes (ii) and (iii) one observes that for typical QD structures ARP works often best for pulse areas given by a few multiples of $\pi$ while the off-resonant preparation requires pulse areas above $10 \pi$. On the other hand, they have an opposite trend when varying the phonon coupling constant, e.g., by considering different material systems. Since phonon emission becomes more efficient for increasing exciton-phonon coupling the pulse area required for the state preparation in scheme (iii) decreases, while the coherent schemes (i) and (ii) typically deteriorate for increasing phonon coupling.

Also the temperature plays a quite different role in the different schemes. Schemes (ii) and (iii) rely on the absence of phonon absorption processes between the dressed states. Since the splitting between the dressed states is in the range of a few $\mathrm{meV}$, already at temperatures of about $20 \mathrm{~K}$ the rate of phonon absorption is of the same order as the phonon emission rate. Therefore, an equal occupation of ground and exciton state will be reached and the state preparation becomes rather inefficient. In the case of ARP this limitation may be partly overcome either by using pulses with rather low pulse areas around $\pi$, where phonon-induced transitions are not yet very efficient or by using pulses with very high pulse areas, where the phonons are effectively decoupled. In the resonant scheme (i), on the other hand, at least a few Rabi rotations have been clearly observed up to temperatures of $50 \mathrm{~K}$. Even higher temperatures should in principle be possible by using shorter pulses, as long as the increased spectral width of the pulse does not lead to the excitation of other nearby transitions which may be present in a real QD.

The above discussion clearly demonstrates that there is not a single simple criterion which defines the "best" state preparation scheme in a QD. Instead, the 
selection of the optimal scheme for a given purpose depends on the final state to be prepared as well as on a variety of different conditions such as material parameters or temperature, but also on limitations due to phenomena not included in the model. Other nearby transitions may set a lower limit for the pulse duration, while additional dephasing or relaxation channels may set an upper limit for the time of the preparation process. However, the discussion also clearly demonstrates that the underlying model, despite its simplicity, is able to quantitatively describe a variety of experimental results and therefore can be seen as a prototypical model for an interacting many-body system which, due to its simplicity, is able to provide detailed physical insights in the dynamics of coupled fermion-boson systems.

\section{Acknowledgments}

This work was supported by the Deutsche Forschungsgemeinschaft DFG through the grant Ax 17/7-1.

[1] Bimberg D, Grundmann M and Ledentsov N 1999 Quantum Dot Heterostructures (Chichester: Wiley)

[2] Schweizer H, Jetter M and Scholz F 2003 Quantum-dot lasers Single Quantum Dots: Fundamentals, Applications and New Concepts ed Michler P (Berlin: Springer) p 185

[3] Ledentsov N N 2011 Semicond. Sci. Technol. 26014001

[4] Chow W W and Jahnke F 2013 Progr. Quantum Electron. 37109

[5] Michler P, Kiraz A, Becher C, Schoenfeld W V, Petroff P M, Zhang L, Hu E and Imamoglu A 2000 Science 2902282

[6] Santori C, Pelton M, Solomon G, Dale Y and Yamamoto Y 2001 Phys. Rev. Lett. 861502

[7] Yuan Z, Kardynal B E, Stevenson R M, Shields A J, Lobo C J, Cooper K, Beattie N S, Ritchie D A and Pepper M 2002 Science 295102

[8] Press D, Götzinger S, Reitzenstein S, Hofmann C, Löffler A, Kamp M, Forchel A and Yamamoto Y 2007 Phys. Rev. Lett. 98117402

[9] Yılmaz S T, Fallahi P and Imamoğlu A 2010 Phys. Rev. Lett. 105033601

[10] Eisaman M D, Fan J, Migdall A and Polyakov S V 2011 Rev. Sci. Instrum. 82071101

[11] Benson O, Santori C, Pelton M and Yamamoto Y 2000 Phys. Rev. Lett. 842513

[12] Moreau E, Robert I, Manin L, Thierry-Mieg V, Gérard J M and Abram I 2001 Phys. Rev. Lett. 87183601

[13] Santori C, Fattal D, Pelton M, Solomon G S and Yamamoto Y 2002 Phys. Rev. B 66045308

[14] Akopian N, Lindner N, Poem E, Berlatzky Y, Avron J, Gershoni D, Gerardot B and Petroff P 2006 Phys. Rev. Lett. 96130501

[15] Stevenson R M, Young R J, Atkinson P, Cooper K, Ritchie D A and Shields A J 2006 Nature 439179

[16] Hafenbrak R, Ulrich S M, Michler P, Wang L, Rastelli A and Schmidt O G 2007 New J. Phys 9315

[17] Dousse A, Suffczyński J, Beveratos A, Krebs O, Lemaître A, Sagnes I, Bloch J, Voisin P and Senellart P 2010 Nature 466217

[18] Müller M, Bounouar S, Jöns K D, Glässl M and Michler P 2014 Nature Photonics 8224

[19] Bonadeo N H, Erland J, Gammon D, Park D, Katzer D S and Steel D G 1998 Science 282 1473

[20] Biolatti E, Iotti R C, Zanardi P and Rossi F 2000 Phys. Rev. Lett. 855647

[21] Troiani F, Hohenester U and Molinari E 2000 Phys. Rev. B 622263

[22] D'Amico I, Biolatti E, Pazy E, Zanardi P and Rossi F 2002 Physica E: Low-dimensional Systems and Nanostructures 13620

[23] Boyle S J, Ramsay A J, Bello F, Liu H Y, Hopkinson M, Fox A M and Skolnick M S 2008 Phys. Rev. B 78075301

[24] Michaelis De Vasconcellos S, Gordon S, Bichler M, Meier T and Zrenner A 2010 Nature Photonics 4545

[25] Ramsay A J 2010 Semicond. Sci. Technol. 25103001

[26] Rossi F (ed) 2005 Semiconductor macroatoms (London: Imperial College Press)

[27] Jacak L, Hawrylak P and Wójs A 1998 Quantum dots (Berlin: Springer)

[28] Paillard M, Marie X, Renucci P, Amand T, Jbeli A and Gerard J M 2001 Phys. Rev. Lett. 86 1634 
[29] Tsitsishvili E, Baltz R v and Kalt H 2005 Phys. Rev. B 72155333

[30] Roszak K, Axt V, Kuhn T and Machnikowski P 2007 Phys. Rev. B 76195324

[31] Poem E, Kodriano Y, Tradonsky C, Lindner N H, Gerardot B D, Petroff P M and Gershoni D 2010 Nature Physics 6993

[32] Langbein W, Borri P, Woggon U, Stavarache V, Reuter D and Wieck A D 2004 Phys. Rev. B 69161301

[33] Seguin R, Schliwa A, Rodt S, Pötschke K, Pohl U W and Bimberg D 2005 Phys. Rev. Lett. 95 257402

[34] Ghali M, Ohtani K, Ohno Y and Ohno H 2012 Nature Communications 3661

[35] Kuroda T, Mano T, Ha N, Nakajima H, Kumano H, Urbaszek B, Jo M, Abbarchi M, Sakuma Y, Sakoda K, Suemune I, Marie X and Amand T 2013 Phys. Rev. B 88041306

[36] Coish W A and Gambetta J M 2009 Phys. Rev. B 80241303

[37] Nicoll C A, Salter C L, Stevenson R M, Hudson A J, Atkinson P, Cooper K, Shields A J and Ritchie D A 2009 J. Cryst. Growth 3111811

[38] Tartakovskii A I, Makhonin M N, Sellers I R, Cahill J, Andreev A D, Whittaker D M, Wells J P R, Fox A M, Mowbray D J, Skolnick M S et al. 2004 Phys. Rev. B 70193303

[39] Schliwa A, Winkelnkemper M, Lochmann A, Stock E and Bimberg D 2009 Phys. Rev. B 80 161307

[40] Muller A, Fang W, Lawall J and Solomon G S 2009 Phys. Rev. Lett. 103217402

[41] Bayer M, Ortner G, Stern O, Kuther A, Gorbunov A A, Forchel A, Hawrylak P, Fafard S, Hinzer K, Reinecke T L, Walck S N, Reithmaier J P, Klopf F and Schäfer F 2002 Phys. Rev. B 65195315

[42] Kowalik K, Krebs O, Lemaitre A, Laurent S, Senellart P, Voisin P and Gaj J A 2005 Appl. Phys. Lett. 861907

[43] Seidl S, Kroner M, Högele A, Karrai K, Warburton R J, Badolato A and Petroff P M 2006 Appl. Phys. Lett. 88203113

[44] Stevenson R M, Young R J, See P, Gevaux D G, Cooper K, Atkinson P, Farrer I, Ritchie D A and Shields A J 2006 Phys. Rev. B 73033306

[45] Trotta R, Zallo E, Ortix C, Atkinson P, Plumhof J D, van den Brink J, Rastelli A and Schmidt O G 2012 Phys. Rev. Lett. 109147401

[46] Welander E and Burkard G 2012 Phys. Rev. B 86165312

[47] Glässl M and Axt V M 2012 Phys. Rev. B 86245306

[48] Mahan G D 2000 Many-Particle Physics (New York: Kluwer)

[49] Takagahara T 1999 Phys. Rev. B 602638

[50] Besombes L, Kheng K, Marsal L and Mariette H 2001 Phys. Rev. B 63155307

[51] Krummheuer B, Axt V M and Kuhn T 2002 Phys. Rev. B 65195313

[52] Vagov A, Axt V M, Kuhn T, Langbein W, Borri P and Woggon U 2004 Phys. Rev. B 70201305

[53] Ramsay A J, Gopal A V, Gauger E M, Nazir A, Lovett B W, Fox A M and Skolnick M S 2010 Phys. Rev. Lett. 10417402

[54] Ramsay A J, Godden T M, Boyle S J, Gauger E M, Nazir A, Lovett B W, Fox A M and Skolnick M S 2010 Phys. Rev. Lett. 105177402

[55] Krummheuer B, Axt V M, Kuhn T, D'Amico I and Rossi F 2005 Phys. Rev. B 71235329

[56] Hodgson T E, Viola L and D'Amico I 2008 Phys. Rev. B 78165311

[57] Hameau S, Guldner Y, Verzelen O, Ferreira R, Bastard G, Zeman J, Lemaître A and Gerard J M 1999 Phys. Rev. Lett. 834152

[58] Carmele A, Richter M, Chow W and Knorr A 2010 Phys. Rev. Lett. 104156801

[59] Schuh K, Seebeck J, Lorke M and Jahnke F 2009 Appl. Phys. Lett. 94201108

[60] Reiter D E, Wigger D, Axt V M and Kuhn T 2011 Phys. Rev. B 84195327

[61] Findeis F, Zrenner A, Böhm G and Abstreiter G 2000 Phys. Rev. B 61 R10579

[62] Stock E, Dachner M, Warming T, Schliwa A, Lochmann A, Hoffmann A, Toropov A, Bakarov A, Derebezov I, Richter M et al. 2011 Phys. Rev. B 83041304

[63] Calarco T, Datta A, Fedichev P, Pazy E and Zoller P 2003 Phys. Rev. A 68012310

[64] McCutcheon D P S and Nazir A 2010 New J. Phys 12113042

[65] Weiss U 2008 Quantum dissipative systems 3rd ed (Singapore: World Scientific)

[66] Nysteen A, Kaer P and Mørk J 2013 Phys. Rev. Lett. 110087401

[67] Ostapenko I, Hönig G, Rodt S, Schliwa A, Hoffmann A, Bimberg D, Dachner M R, Richter M, Knorr A, Kako S et al. 2012 Phys. Rev. B 85081303

[68] Gross F and Zimmermann R 2007 Phys. Rev. B 75235320

[69] Krummheuer B, Axt V M and Kuhn T 2005 Phys. Rev. B 72245336

[70] Debald S, Brandes T and Kramer B 2002 Phys. Rev. B 66041301

[71] Lindwall G, Wacker A, Weber C and Knorr A 2007 Phys. Rev. Lett. 99087401 
[72] Rozbicki E and Machnikowski P 2008 Phys. Rev. Lett. 10027401

[73] Gawarecki K, Pochwała M, Grodecka-Grad A and Machnikowski P 2010 Phys. Rev. B 81 245312

[74] Allen L and Eberly J H 1975 Optical Resonance and Two-Level Atoms (New York: John Wiley and Sons)

[75] Huang K and Rhys A 1950 Proc. Roy. Soc. London, Ser. A 204406

[76] Duke C B and Mahan G D 1965 Phys. Rev. 139 A1965

[77] Schmitt-Rink S, Miller D A B and Chemla D S 1987 Phys. Rev. B 358113

[78] Borri P, Langbein W, Schneider S, Woggon U, Sellin R L, Ouyang D and Bimberg D 2001 Phys. Rev. Lett. 87157401

[79] Bayer M and Forchel A 2002 Phys. Rev. B 65041308

[80] Langbein W, Borri P, Woggon U, Stavarache V, Reuter D and Wieck A D 2004 Phys. Rev. B $\mathbf{7 0} 033301$

[81] Chang W H, Chen W Y, Chang H S, Hsieh T P, Chyi J I and Hsu T M 2006 Phys. Rev. Lett. 96117401

[82] Ates S, Ulrich S M, Ulhaq A, Reitzenstein S, Löffler A, Höfling S, Forchel A and Michler P 2009 Nature Photonics 3724

[83] Brüggemann C, Akimov A V, Scherbakov A V, Bombeck M, Schneider C, Höfling S, Forchel A, Yakovlev D R and Bayer M 2011 Nature Photonics 630

[84] Kasprzak J, Sivalertporn K, Albert F, Schneider C, Höfling S, Kamp M, Forchel A, Reitzenstein S, Muljarov E A and Langbein W 2013 New J. Phys 15045013

[85] Jakubczyk T, Pacuski W, Smoleński T, Golnik A, Florian M, Jahnke F, Kruse C, Hommel D and Kossacki P 2013 J. Appl. Phys. 113136504

[86] Blattmann R, Krenner H J, Kohler S and Hänggi P 2014 Phys. Rev. A 89012327

[87] Kaer P, Nielsen T R, Lodahl P, Jauho A P and Mørk J 2010 Phys. Rev. Lett. 104157401

[88] Roy C and Hughes S 2011 Phys. Rev. Lett. 106247403

[89] Roy C and Hughes S 2011 Phys. Rev. X 1021009

[90] Kaer P, Nielsen T R, Lodahl P, Jauho A P and Mørk J 2012 Phys. Rev. B 86085302

[91] Glässl M, Sörgel L, Vagov A, Croitoru M D, Kuhn T and Axt V M 2012 Phys. Rev. B 86 035319

[92] Nazir A 2008 Phys. Rev. B 78153309

[93] Harsij Z, Bagheri Harouni M, Roknizadeh R and Naderi M H 2012 Phys. Rev. A 86063803

[94] Rastghalam S A and Harouni M B 2013 LASER PHYSICS 23115202

[95] Kaer P, Lodahl P, Jauho A P and Mork J 2013 Phys. Rev. B 87081308

[96] Naesby A, Suhr T, Kristensen P T and Mørk J 2008 Phys. Rev. A 78045802

[97] Hohenester U, Laucht A, Kaniber M, Hauke N, Neumann A, Mohtashami A, Seliger M, Bichler M and Finley J J 2009 Phys. Rev. B 80201311

[98] Hohenester U 2010 Phys. Rev. B 81155303

[99] Calic M, Gallo P, Felici M, Atlasov K A, Dwir B, Rudra A, Biasiol G, Sorba L, Tarel G, Savona V and Kapon E 2011 Phys. Rev. Lett. 106227402

[100] Majumdar A, Kim E, Gong Y, Bajcsy M and Vučković J 2011 Phys. Rev. B 84085309

[101] Hughes S, Yao P, Milde F, Knorr A, Dalacu D, Mnaymneh K, Sazonova V, Poole P, Aers G, Lapointe J et al. 2011 Phys. Rev. B 83165313

[102] Ulrich S M, Ates S, Reitzenstein S, Löffler A, Forchel A and Michler P 2011 Phys. Rev. Lett. 106247402

[103] Bayer M, Hawrylak P, Hinzer K, Fafard S, Korkusinski M, Wasilewski Z R, Stern O and Forchel A 2001 Science 291451

[104] Krenner H J, Sabathil M, Clark E C, Kress A, Schuh D, Bichler M, Abstreiter G and Finley J J 2005 Phys. Rev. Lett. 94057402

[105] Stinaff E A, Scheibner M, Bracker A S, Ponomarev I V, Korenev V L, Ware M E, Doty M F, Reinecke T L and Gammon D 2006 Science 311636

[106] Gywat O, Burkard G and Loss D 2002 Phys. Rev. B 65205329

[107] Scheibner M, Ponomarev I V, Stinaff E A, Doty M F, Bracker A S, Hellberg C S, Reinecke T L and Gammon D 2007 Phys. Rev. Lett. 99197402

[108] Muljarov E A, Takagahara T and Zimmermann R 2005 Phys. Rev. Lett. 95177405

[109] Gawarecki K and Machnikowski P 2012 Phys. Rev. B 85 041305(R)

[110] Daniels J M, Machnikowski P and Kuhn T 2013 Phys. Rev. B 88205307

[111] López-Richard V, Oliveira S S and Hai G Q 2005 Phys. Rev. B 71075329

[112] Müller K, Bechtold A, Ruppert C, Zecherle M, Reithmaier G, Bichler M, Krenner H J, Abstreiter G, Holleitner A W, Villas-Boas J M, Betz M and Finley J J 2012 Phys. Rev. Lett. 108197402 
[113] Kerfoot M L, Govorov A O, Czarnocki C, Lu D, Gad Y N, Bracker A S, Gammon D and Scheibner M 2014 Nature Communications 53299

[114] Huneke J, Krügel A, Kuhn T, Vagov A and Axt V M 2008 Phys. Rev. B 7885316

[115] Roszak K and Machnikowski P 2006 Phys. Rev. A 73022313

[116] Breuer H P and Petruccione F 2002 The theory of open quantum systems (Oxford: Oxford University Press)

[117] Leggett A J, Chakravarty S, Dorsey A T, Fisher M P A, Garg A and Zwerger W 1987 Rev. Mod. Phys. 591

[118] Vagov A, Axt V M and Kuhn T 2002 Phys. Rev. B 66165312

[119] Axt V M, Kuhn T, Vagov A and Peeters F M 2005 Phys. Rev. B 72125309

[120] Machnikowski P 2008 Phys. Rev. B 78195320

[121] Gawarecki K, Lüker S, Reiter D E, Kuhn T, Glässl M, Axt V M, Grodecka ${ }^{\circ}$ Grad A and Machnikowski P 2012 Phys. Rev. B 86235301

[122] Rossi F and Kuhn T 2002 Rev. Mod. Phys. 74895

[123] Förstner J, Weber C, Danckwerts J and Knorr A 2003 Phys. Rev. Lett. 91127401

[124] Hohenester U and Stadler G 2004 Phys. Rev. Lett. 92196801

[125] Krügel A, Axt V M, Kuhn T, Machnikowski P and Vagov A 2005 Appl. Phys. B 81897

[126] Krügel A, Axt V M and Kuhn T 2006 Phys. Rev. B 73035302

[127] McCutcheon D P S, Dattani N S, Gauger E M, Lovett B W and Nazir A 2011 Phys. Rev. B 84081305

[128] Roy C and Hughes S 2012 Phys. Rev. B 85115309

[129] Machnikowski P and Jacak L 2004 Phys. Rev. B 69193302

[130] Vagov A, Croitoru M D, Axt V M, Kuhn T and Peeters F M 2006 Phys. Status Solidi B 243 2233

[131] Vagov A, Croitoru M D, Axt V M, Kuhn T and Peeters F M 2007 Phys. Rev. Lett. 98227403

[132] Vagov A, Croitoru M, Glässl M, Axt V and Kuhn T 2011 Phys. Rev. B 83094303

[133] Thorwart M, Eckel J and Mucciolo E R 2005 Phys. Rev. B 72235320

[134] Glässl M, Croitoru M D, Vagov A, Axt V M and Kuhn T 2012 Phys. Rev. B 85195306

[135] Glässl M, Vagov A, Lüker S, Reiter D E, Croitoru M D, Machnikowski P, Axt V M and Kuhn T 2011 Phys. Rev. B 84195311

[136] Vagov A, Croitoru M D, Axt V M, Machnikowski P and Kuhn T 2011 Phys. Status Solidi B 248839

[137] Förstner J, Ahn K J, Danckwerts J, Schaarschmidt M, Waldmuller I, Weber C and Knorr A 2002 Phys. Status Solidi B 234155

[138] Rabi I I 1937 Phys. Rev. 51652

[139] Boyle S J, Ramsay A J, Fox A M, Skolnick M S, Heberle A P and Hopkinson M 2009 Phys. Rev. Lett. 102207401

[140] Muller A, Flagg E B, Bianucci P, Wang X Y, Deppe D G, Ma W, Zhang J, Salamo G J, Xiao M and Shih C K 2007 Phys. Rev. Lett. 99187402

[141] Xu X, Sun B, Berman P R, Steel D G, Bracker A S, Gammon D and Sham L J 2007 Science $\mathbf{3 1 7} 929$

[142] Flagg E B, Muller A, Robertson J W, Founta S, Deppe D G, Xiao M, Ma W, Salamo G J and Shih C K 2009 Nature Physics 5203

[143] Schaibley J R, Burgers A P, McCracken G A, Steel D G, Bracker A S, Gammon D and Sham L J 2013 Phys. Rev. B 87115311

[144] Akimov A V, Scherbakov A V, Yakovlev D R, Foxon C T and Bayer M 2006 Phys. Rev. Lett. 97037401

[145] Gotoh H, Sanada H, Yamaguchi H and Sogawa T 2013 Applied Physics Letters 103112104

[146] Metcalfe M, Carr S M, Muller A, Solomon G S and Lawall J 2010 Phys. Rev. Lett. 105037401

[147] Borri P, Langbein W, Schneider S, Woggon U, Sellin R L, Ouyang D and Bimberg D 2002 Phys. Rev. B 66 081306(R)

[148] Zecherle M, Ruppert C, Clark E C, Abstreiter G, Finley J J and Betz M 2010 Phys. Rev. B 82(12) 125314

[149] Stufler S, Machnikowski P, Ester P, Bichler M, Axt V M, Kuhn T and Zrenner A 2006 Phys. Rev. B 73125304

[150] Bensky G, Nair S V, Ruda H E, Dasgupta S, Kurizki G and Brumer P 2013 J. Phys. B 46 055503

[151] Sun E, Feng D and Jia T 2014 Adv. Cond. Mat. Phys. 73219381

[152] Stievater T H, Li X, Steel D G, Gammon D, Katzer D S, Park D, Piermarocchi C and Sham L J 2001 Phys. Rev. Lett. 87133603

[153] Kamada H, Gotoh H, Temmyo J, Takagahara T and Ando H 2001 Phys. Rev. Lett. 87246401 
[154] Htoon H, Takagahara T, Kulik D, Baklenov O, Holmes A L and Shih C K 2002 Phys. Rev. Lett. $\mathbf{8 8} 087401$

[155] Zrenner A, Beham E, Stufler S, Findeis F, Bichler M and Abstreiter G 2002 Nature 418612

[156] Besombes L, Baumberg J J and Motohisa J 2003 Phys. Rev. Lett. 90257402

[157] Stufler S, Ester P, Zrenner A and Bichler M 2005 Phys. Rev. B 72121301

[158] Wang Q Q, Muller A, Bianucci P, Rossi E, Xue Q K, Takagahara T, Piermarocchi C, MacDonald A H and Shih C K 2005 Phys. Rev. B 72035306

[159] Kuroda K, Kuroda T, Watanabe K, Mano T, Sakoda K, Kido G and Koguchi N 2007 Appl. Phys. Lett. 90051909

[160] Melet R, Voliotis V, Enderlin A, Roditchev D, Wang X L, Guillet T and Grousson R 2008 Phys. Rev. B $\mathbf{7 8} 073301$

[161] Takagi H, Nakaoka T, Watanabe K, Kumagai N and Arakawa Y 2008 Optics Express 1613949

[162] Ravaro M, Enderlin A, Tonin C, Voliotis V, Grousson R, Lemaitre A and Martinez A $2010 \mathrm{~J}$. Phys.: Conf. Ser. 245012053

[163] Wolpert C, Dicken C, Wang L, Atkinson P, Rastelli A, Schmidt O G, Giessen H and Lippitz M 2012 physica status solidi (b) 249721

[164] Monniello L, Tonin C, Hostein R, Lemaitre A, Martinez A, Voliotis V and Grousson R 2013 Phys. Rev. Lett. 111026403

[165] Li X, Wu Y, Steel D, Gammon D, Stievater T H, Katzer D S, Park D and Piermarocchi C and Sham L J 2003 Science 301809

[166] Flissikowski T, Betke A, Akimov I and Henneberger F 2004 Phys. Rev. Lett. 92227401

[167] Jayakumar H, Predojević A, Huber T, Kauten T, Solomon G S and Weihs G 2013 Phys. Rev. Lett. 110135505

[168] Boyle S J, Ramsay A J, Fox A M and Skolnick M S 2010 Physica E 422485

[169] Wu Z J, Zhu K D and Zheng H 2004 Phys. Lett. A 333310

[170] Glässl M, Croitoru M D, Vagov A, Axt V M and Kuhn T 2011 Phys. Rev. B 84125304

[171] Reiter D E, Lüker S, Gawarecki K, Grodecka-Grad A, Machnikowski P, Axt V M and Kuhn T 2012 Acta Phys. Pol. A 1221065

[172] Mogilevtsev D, Nisovtsev A P, Kilin S, Cavalcanti S B, Brandi H S and Oliveira L E 2008 Phys. Rev. Lett. 100017401

[173] Gerry C C and Knight P L 2005 Introductory Quantum Optics (Cambridge: Cambridge University Press)

[174] Wigger D, Lüker S, Reiter D E, Axt V M, Machnikowski P and Kuhn T 2014 J. Phys. Condens. Matter 26255802

[175] Alicki R, Horodecki M, Horodecki P, Horodecki R, Jacak L and Machnikowski P 2004 Phys. Rev. A 70010501

[176] Reithmaier J P, Sẹk G, Löffler A, Hofmann C, Kuhn S, Reitzenstein S, Keldysh L V, Kulakovskii V D, Reinecke T L and Forchel A 2004 Nature 432197

[177] Yoshie T, Scherer A, Hendrickson J, Khitrova G, Gibbs H M, Rupper G, Ell C, Shchekin O B and Deppe D G 2004 Nature 432200

[178] Peter E, Senellart P, Martrou D, Lemaître A, Hours J, Gérard J M and Bloch J 2005 Phys. Rev. Lett. 95067401

[179] Hennessy K, Badolato A, Winger M, Gerace D, Atatüre M, Gulde S, Fält S, Hu E L and Imamoglu A 2007 Nature 445896

[180] Ulhaq A, Ates S, Weiler S, Ulrich S M, Reitzenstein S, Löffler A, Höfling S, Worschech L, Forchel A and Michler P 2010 Phys. Rev. B 82045307

[181] Zhu K D and Li W S 2003 Phys. Lett. A 314380

[182] Roy C, Kim H, Waks E and Hughes S 2012 Phot. Nano. Fund. Appl. 10359

[183] Nielsen M A and Chuang I L 2000 Quantum Computation and Quantum Information (Cambridge: Cambridge University Press)

[184] Stufler S, Ester P, Zrenner A and Bichler M 2006 Phys. Rev. Lett. 96037402

[185] Roszak K and Machnikowski P 2006 Phys. Lett. A 351251

[186] Wigger D, Reiter D E, Axt V M and Kuhn T 2013 Phys. Rev. B 87085301

[187] Borri P and W L 2007 J. Phys. Condens. Matter 19295201

[188] Patton B, Langbein W, Woggon U, Maingault L and Mariette H 2006 Phys. Rev. B 73235354

[189] Kasprzak J, Patton B, Savona V and Langbein W 2011 Nature Photon. 557

[190] Kasprzak J, Portolan S, Rastelli A, Wang L, Plumhof J D, Schmidt O G and Langbein W 2013 New J. Phys 15055006

[191] Axt V M, Machnikowski P and Kuhn T 2005 Phys. Rev. B 71155305

[192] Machnikowski P, Axt V M and Kuhn T 2007 Phys. Rev. A 75052330

[193] Malinovsky V S and Krause J L 2001 Eur. Phys. J. D 14147 
[194] Melinger J S, Gandhi S R, Hariharan A, Goswami D and Warren W S 1994 J. Chem. Phys. 1016439

[195] Saleh B E A and Teich M C 2007 Fundamentals of photonics Wiley series in pure and applied optics (New York: Wiley-Interscience)

[196] Wu Y, Piper I M, Ediger M, Brereton P, Schmidgall E R, Eastham P R, Hugues M, Hopkinson M and Phillips R T 2011 Phys. Rev. Lett. 106067401

[197] Simon C M, Belhadj T, Chatel B, Amand T, Renucci P, Lemaitre A, Krebs O, Dalgarno P A, Warburton R J, Marie X and Urbaszek B 2011 Phys. Rev. Lett. 106166801

[198] Debnath A, Meier C, Chatel B and Amand T 2012 Phys. Rev. B 86 161304(R)

[199] Vitanov N V, Halfmann T, Shore B W and Bergmann K 2001 Annu. Rev. Phys. Chem. 52763

[200] Tannor D J 2007 Introduction to quantum mechanics (Sausalito,California: University Science Books)

[201] Zener C 1932 Proc. R. Soc. A 137696

[202] Landau L D 1932 Phys. Z. Sowjet. 188

[203] Schmidgall E R, Eastham P R and Phillips R T 2010 Phys. Rev. B 81195306

[204] Lüker S, Gawarecki K, Reiter D E, Grodecka-Grad A, Axt V M, Machnikowski P and Kuhn T 2012 Phys. Rev. B 85121302

[205] Eastham P R, Spracklen A O and Keeling J 2013 Phys. Rev. B 87195306

[206] Mathew R, Dilcher E, Gamouras A, Ramachandran A, Yang H Y S, Freisem S, Deppe D and Hall K C 2014 Phys. Rev. B 90035316

[207] Glässl M, Barth A M, Gawarecki K, Machnikowski P, Croitoru M D, Lüker S, Reiter D E, Kuhn T and Axt V M 2013 Phys. Rev. B 87085303

[208] Hui H Y and Liu R B 2008 Phys. Rev. B 78155315

[209] Debnath A, Meier C, Chatel B and Amand T 2013 Phys. Rev. B 88 201305(R)

[210] Ramsay A J, Godden T M, Boyle S J, Gauger E M, Nazir A, Lovett B W, Gopal A, Fox A M and Skolnick M S 2011 J. Appl. Phys. 109102415

[211] Glässl M, Barth A M and Axt V M 2013 Phys. Rev. Lett. 110147401

[212] Hughes S and Carmichael H J 2013 New J. Phys 15053039

[213] Reithmaier J P 2008 Semicond. Sci. Technol. 23123001 